\title{
MAXIMIZATION OF THE PORTFOLIO GROWTH RATE UNDER FIXED AND PROPORTIONAL TRANSACTION COSTS
}

\author{
JAN PALCZEWSKI* AND ŁUKASZ STETTNER ${ }^{\dagger}$
}

\begin{abstract}
This paper considers a discrete-time Markovian model of asset prices with economic factors and transaction costs with proportional and fixed terms. Existence of optimal strategies maximizing average growth rate of portfolio is proved in the case of complete and partial observation of the process modelling the economic factors. The proof is based on a modification of the vanishing discount approach. The main difficulty is the discontinuity of the controlled transition operator of the underlying Markov process.
\end{abstract}

Key words: portfolio optimization, growth rate, transaction costs, incomplete information, Markov process, impulsive strategy, optimal control, vanishing discount

1. Introduction. On a given probability space $(\Omega, \mathcal{F}, \mathbb{P})$ with discrete filtration $\left(\mathcal{F}_{t}\right)_{t=0,1, \ldots}$, where $\mathcal{F}_{0}$ is trivial, consider a market model driven by a time homogeneous Markov process $(S(t), Z(t))_{t=0,1, \ldots}$, where $S(t)=\left(S^{1}(t), \ldots, S^{d}(t)\right) \in(0, \infty)^{d}$ denotes prices of $d$ financial assets and $Z(t) \in(E, \mathcal{E})$, where $E$ is a locally compact separable metric space with Borel $\sigma$-algebra $\mathcal{E}$, stands for economic factors. Models with economic factors have been gaining popularity in financial mathematics recently although it has been noted that they add substantially to the complicacy of mathematical methods required for their analysis as compared to models without factors (see eg. [3], [4], [13], [23]). A main advantage of models with economic factors lies in the fact that economic factors can influence market trends therefore change the long-term behaviour of prices. They answer the main criticism of pure Markovian models related to the lack of memory of price processes. Moreover, it is known that models with economic factors allow for better calibration to market data (see [4]).

In the above model, under transaction costs consisting of proportional and constant terms, we maximize the functional

$$
J(\Pi)=\liminf _{T \rightarrow \infty} \frac{1}{T} \mathbb{E} \ln X^{\Pi}(T),
$$

where $X^{\Pi}(T)$ is the wealth of the portfolio (trading strategy) $\Pi$ at time $T$. This functional computes an average growth rate of the portfolio $\Pi$. Indeed, (1) can be

\footnotetext{
${ }^{*}$ Faculty of Mathematics, Warsaw University, Banacha 2, 02-097 Warszawa, Poland, and School of Mathematics, University of Leeds, Leeds LS2 9JT, UK, E-mail: J.Palczewski@mimuw.edu.pl

$\dagger$ Institute of Mathematics, Polish Academy of Sciences, Sniadeckich 8, 00-950 Warszawa, Poland, E-mail: stettner@impan.gov.pl. Research supported by MNiSzW grant 1 P03A 01328.
} 
rewritten as

$$
J(\Pi)=\liminf _{T \rightarrow \infty} \frac{1}{T} \mathbb{E} \sum_{k=0}^{T-1} \ln \frac{X^{\Pi}(k+1)}{X^{\Pi}(k)},
$$

where $\ln \frac{X^{\Pi}(k+1)}{X^{11}(k)}$ is a continuously compounded rate of return in time interval $[k, k+1]$. Functionals of the form (2) are known as long-run average cost functionals. They have been widely studied in the context of stochastic control of Markov processes (see [2], [18], [20] and references therein). Financial applications require, however, additional constraints on admissible controls and give rise to a new class of control problems (see [1], [8], [11], [12], [14], [23] for growth-rate optimization problems on finite and infinite time horizons).

The main result of this paper states that under very general assumptions on the process driving the market there exists an optimal Markovian control for the functional (1). This result is proved by a modification of a vanishing discount approach, as considered in [18], which leads to a certain Bellman inequality. Main difficulties arise from discontinuity of the controlled transition operator of the underlying Markov process, due to a constant term in the transaction costs structure. The above result is not only valid in the case when economic factors are completely observed, but also in models in which economic factors cannot be perfectly read. We also show that the optimal strategy maximizing long run average portfolio growth rate in the case of fixed plus proportional transaction costs is also optimal in the case of proportional transaction costs.

The results obtained in this paper are new in the case of fixed plus proportional transaction costs. They extend application of a general theory of stochastic control to financial problems with a constant term in the transaction cost structure. Moreover, they generalize [1], [23] in the case of only proportional transaction costs.

The paper is organized as follows. In Section 2 we set up a financial model, derive its basic properties and introduce notation. Section 3 presents main results of the paper followed by discussion and remarks. The proof of the main result is contained in Section 4. The case with incomplete observation of the economic factor process is considered in Section 5.

2. Preliminaries. In this section we specify the model in full detail and introduce necessary notation. The dynamics of the price process is governed by

$$
\frac{S^{i}(t+1)}{S^{i}(t)}=\zeta^{i}(Z(t+1), \xi(t+1)), \quad S^{i}(0)=s^{i}>0, \quad i=1, \ldots, d,
$$

where $(\xi(t))_{t=1,2, \ldots}$ is a sequence of i.i.d. random variables with values in a Polish space $\left(E^{\xi}, \mathcal{E}^{\xi}\right)$ and functions $\zeta^{i}:(E, \mathcal{E}) \times\left(E^{\xi}, \mathcal{E}^{\xi}\right) \rightarrow(0, \infty)$ are Borel measurable, $i=$ $1, \ldots, d$. The process $Z(t)$ is a time-homogeneous Markov process. We assume that 
$(S(t), Z(t))_{t=0,1, \ldots}$ is a Feller Markov process, i.e. its transition operator transforms the space bounded continuous functions into itself. We shall write $\zeta^{i}(t)$ for $\zeta^{i}(Z(t+$ $1), \xi(t+1))$ whenever it does not lead to ambiguity. We denote by $\zeta(t)$ the vector $\left(\zeta^{1}(t), \ldots, \zeta^{d}(t)\right)$.

Denote by $\left(\mathcal{F}_{t}^{z}\right)_{t=0,1, \ldots}$ the filtration, where $\mathcal{F}_{0}^{z}$ is a trivial $\sigma$-algebra and $\left(\mathcal{F}_{t}^{z}\right)_{t=1,2, \ldots}$ is generated by the process $(\xi(t), Z(t))_{t=1,2, \ldots}$ with $Z(0)=z$. Notice that the filtration generated by the process $(S(t), Z(t))_{t=0,1, \ldots}$ starting from $(s, z) \in(0, \infty)^{d} \times E$ is identical to $\left(\mathcal{F}_{t}^{z}\right)_{t=0,1, \ldots}$, since it is independent of the initial value of asset prices:

$$
S^{i}(t)=S^{i}(0) \prod_{s=1}^{t} \zeta^{i}(Z(s), \xi(s))
$$

Fix initial values $(s, z)$ for the process $(S(t), Z(t))_{t=0,1, \ldots}$. A trading strategy is a sequence of pairs $\left(\left(N_{k}, \tau_{k}\right)\right)_{k=1,2, \ldots}$, where $\tau_{k}$ is an $\left(\mathcal{F}_{t}^{z}\right)$-stopping time, $\tau_{k+1}>$ $\tau_{k}, k=1,2, \ldots$, and $N_{k}$ is $\mathcal{F}_{\tau_{k}}^{z}$-measurable random variable with values in $[0, \infty)^{d}$ representing the number of shares held in portfolio in the time interval $\left[\tau_{k}, \tau_{k+1}\right)$. By $N(0)$ we denote a deterministic initial portfolio and we set $\tau_{0}=0$. The share holding process at time $t$ is given by

$$
N(t)=\sum_{k=1}^{\infty} 1_{t \in\left[\tau_{k}, \tau_{k+1}\right)} N_{k}
$$

In what follows we shall consider transaction costs of the form

$$
\tilde{c}\left(N_{1}, N_{2}, S\right)=\sum_{i=1}^{d}\left(c_{i}^{1} S^{i}\left(N_{1}^{i}-N_{2}^{i}\right)^{+}+c_{i}^{2} S^{i}\left(N_{1}^{i}-N_{2}^{i}\right)^{-}\right)+c,
$$

where $S$ stands for asset prices, $N_{1}$ denotes portfolio contents before transaction, $N_{2}$ - after transaction, and $c$ is the constant cost charged independently of the size of transaction. Proportional transaction costs are divided into two parts: $c_{i}^{1} \in[0,1)$ is a proportion of the transaction volume paid on buying of asset $i$, while $c_{i}^{2} \in[0,1)$ is applied on selling of asset $i$. We assume that portfolios are self-financing, i.e.

$$
N_{k} \cdot S\left(\tau_{k}\right)=N_{k-1} \cdot S\left(\tau_{k}\right)+\tilde{c}\left(N_{k-1}, N_{k}, S\left(\tau_{k}\right)\right), \quad k=1,2, \ldots
$$

In the case of no transaction costs or proportional transactions costs it is natural to reformulate the problem in terms of proportions. We will also benefit here from this reformulation. Let

$$
\begin{aligned}
& X(t)=N(t) \cdot S(t), \\
& X_{-}(t)=N(t-1) \cdot S(t) .
\end{aligned}
$$

Hence, $X_{-}(t)$ is the wealth of the portfolio before possible transaction at $t$, and $X(t)$ is the wealth just after the transaction. If there is no transaction at $t$ both values are 
identical. In a similar way we construct two processes representing proportions:

$$
\begin{aligned}
& \pi^{i}(t)=\frac{N^{i}(t) S^{i}(t)}{X(t)} \\
& \pi_{-}^{i}(t)=\frac{N^{i}(t-1) S^{i}(t)}{X_{-}(t)}
\end{aligned}
$$

for $i=1,2, \ldots, d$. Since short sales are prohibited we have $\pi(t), \pi_{-}(t) \in \mathcal{S}$, where

$$
\mathcal{S}=\left\{\left(\pi^{1}, \ldots, \pi^{d}\right): \quad \pi^{i} \geq 0, \quad \sum_{i=1}^{d} \pi^{i}=1\right\}
$$

Denote by $\mathcal{S}^{0}$ the simplex $\mathcal{S}$ with its interior

$$
\mathcal{S}^{0}=\left\{\left(\pi^{1}, \ldots, \pi^{d}\right): \quad \pi^{i} \geq 0, \quad \sum_{i=1}^{d} \pi^{i} \leq 1\right\}
$$

and let $g: \mathcal{S}^{0} \rightarrow \mathcal{S}$ be the projection to the boundary

$$
g\left(\pi^{1}, \ldots, \pi^{d}\right)=\left(\frac{\pi^{1}}{\sum \pi^{i}}, \ldots, \frac{\pi^{d}}{\sum \pi^{i}}\right) .
$$

The self-financing condition can be written as

$$
X_{-}\left(\tau_{k}\right)=X\left(\tau_{k}\right)+X_{-}\left(\tau_{k}\right)\left(c\left(\pi_{-}\left(\tau_{k}\right), \tilde{\pi}_{k}\right)+\frac{c}{X_{-}\left(\tau_{k}\right)}\right), \quad k=1,2, \ldots
$$

for some $\tilde{\pi}_{k} \in \mathcal{S}^{0}$ such that $\pi\left(\tau_{k}\right)=g\left(\tilde{\pi}_{k}\right)$ and

$$
c\left(\pi_{-}, \tilde{\pi}\right)=\sum_{i=1}^{d}\left(c_{i}^{1}\left(\tilde{\pi}^{i}-\pi_{-}^{i}\right)^{+}+c_{i}^{2}\left(\tilde{\pi}^{i}-\pi_{-}^{i}\right)^{-}\right)
$$

is the proportion of the portfolio wealth that is consumed by proportional part of transaction costs. From (5) one can deduce that $\tilde{\pi}_{k}=\frac{X\left(\tau_{k}\right)}{X_{-}\left(\tau_{k}\right)} \pi\left(\tau_{k}\right)$ fulfills (8). We shall show that this is a unique solution to (8). Given $\pi_{-}, \pi \in \mathcal{S}, x_{-}>0$ define a function

$$
\tilde{F}^{\pi_{-}, \pi, x_{-}}(\delta)=c\left(\pi_{-}, \delta \pi\right)+\frac{c}{x_{-}}+\delta .
$$

Notice that (8) is equivalent to

$$
\tilde{F}^{\pi_{-}\left(\tau_{k}\right), \pi\left(\tau_{k}\right), X_{-}\left(\tau_{k}\right)}\left(\sum_{i=1}^{d} \tilde{\pi}_{k}^{i}\right)=1 .
$$

It can be proved (see [23]) that there exists a unique function $\tilde{e}: \mathcal{S} \times \mathcal{S} \times(0, \infty) \rightarrow[0,1]$ such that

$$
\tilde{F}^{\pi_{-}, \pi, x_{-}}\left(\tilde{e}\left(\pi_{-}, \pi, x_{-}\right)\right)=1
$$


if $\tilde{F}^{\pi_{-}, \pi, x_{-}}(\delta)=1$ has a solution $\delta \in(0,1]$ and $\tilde{e}\left(\pi_{-}, \pi, x_{-}\right)=0$, otherwise (the wealth of the portfolio is too small to perform requested change of proportions). For technical reasons this is an undesirable condition. Therefore, we shall modify transaction costs in such a way that the transaction is possible at any moment. Let

$$
x^{*}>\frac{c}{1-\max _{i} c_{i}^{h}}, \quad h=1,2,
$$

and modify (4) in the following way

$$
\begin{gathered}
\tilde{c}\left(N_{1}, N_{2}, S\right)=\sum_{i=1}^{d}\left(c_{i}^{1} S^{i}\left(N_{1}^{i}-N_{2}^{i}\right)^{+}+c_{i}^{2} S^{i}\left(N_{1}^{i}-N_{2}^{i}\right)^{-}\right) \\
+ \begin{cases}c, & \text { when } N_{1} \cdot S \geq x^{*}, \\
c \frac{N_{1} \cdot S}{x^{*}}, & \text { when } N_{1} \cdot S<x^{*} .\end{cases}
\end{gathered}
$$

Notice that for portfolios with the wealth over $x^{*}$ usual constant plus proportional transaction costs are applied as in (4). Transaction costs are modified only for wealth below $x^{*}$, when the constant cost is replaced by appropriate proportional term. It is not restrictive in practical applications where portfolio wealth is counted in thousands of dollars. As an example consider fixed cost of 1 USD and proportional cost $-0.5 \%$. We obtain from (9) that $x^{*}>1.0051$ USD.

With the new transaction costs structure the self-financing condition (8) takes the form

$$
X_{-}\left(\tau_{k}\right)=X\left(\tau_{k}\right)+X_{-}\left(\tau_{k}\right)\left(c\left(\pi_{-}\left(\tau_{k}\right), \tilde{\pi}_{k}\right)+\frac{c}{X_{-}\left(\tau_{k}\right) \vee x^{*}}\right), \quad k=1,2, \ldots
$$

where $a \vee b=\max (a, b)$ and $\tilde{\pi}_{k}=\frac{X\left(\tau_{k}\right)}{X_{-}\left(\tau_{k}\right)} \pi\left(\tau_{k}\right)$. Given $\pi_{-}, \pi \in \mathcal{S}, x_{-}>0$ we define a function

$$
F^{\pi_{-}, \pi, x_{-}}(\delta)=c\left(\pi_{-}, \delta \pi\right)+\frac{c}{x_{-} \vee x^{*}}+\delta .
$$

The above self-financing condition is equivalent to

$$
F^{\pi_{-}\left(\tau_{k}\right), \pi\left(\tau_{k}\right), X_{-}\left(\tau_{k}\right)}\left(\sum_{i=1}^{d} \tilde{\pi}_{k}^{i}\right)=1
$$

Lemma 2.1. There exists a unique function e $: \mathcal{S} \times \mathcal{S} \times(0, \infty) \rightarrow(0,1]$, such that

$$
F^{\pi_{-}, \pi, x_{-}}\left(e\left(\pi_{-}, \pi, x_{-}\right)\right)=1
$$

Moreover, $e$ is continuous and $\inf e\left(\pi_{-}, \pi, x_{-}\right)>0$.

Proof. The proof is rather straightforward and resembles the proof of Lemma $1 \mathrm{in}$ [23]. 
The uniqueness of the function $e$ implies that $\frac{X\left(\tau_{k}\right)}{X_{-}\left(\tau_{k}\right)}=e\left(\pi_{-}\left(\tau_{k}\right), \pi\left(\tau_{k}\right), X_{-}\left(\tau_{k}\right)\right)$. Therefore, any transaction can be described solely by means of proportions. Given a pre-transaction wealth $X_{-}\left(\tau_{k}\right)$ and proportions $\pi_{-}\left(\tau_{k}\right)$ at time $\tau_{k}$ one chooses any post-transaction proportions $\pi\left(\tau_{k}\right) \in \mathcal{S}$. As a result the wealth is diminished to

$$
X\left(\tau_{k}\right)=X_{-}\left(\tau_{k}\right) e\left(\pi_{-}\left(\tau_{k}\right), \pi\left(\tau_{k}\right), X_{-}\left(\tau_{k}\right)\right)
$$

Furthermore,

$$
X_{-}(t+1)=\sum_{i=1}^{d} \frac{\pi^{i}(t) X^{i}(t)}{S^{i}(t)} S^{i}(t+1)=X(t)(\pi(t) \cdot \zeta(t)) .
$$

Therefore,

$$
X_{-}(t)=X_{-}(0) \prod_{s=0}^{t-1}(\pi(s) \cdot \zeta(s)) \prod_{k=1}^{\infty}\left(1_{\tau_{k}<t} e\left(\pi_{-}\left(\tau_{k}\right), \pi\left(\tau_{k}\right), X_{-}\left(\tau_{k}\right)\right)+1_{\tau_{k} \geq t}\right)
$$

and the wealth of the portfolio is independent of initial prices of the assets. Therefore, instead of writing $\mathbb{P}^{(s, z)}$ and $\mathbb{E}^{(s, z)}$, it suffices to stress the dependence of the probability measure on the initial condition of the Markov process $(Z(t))$ by writing $\mathbb{P}^{z}$ and $\mathbb{E}^{z}$.

For a given initial value $z \in E$, we say that a sequence $\Pi=\left(\left(\pi_{1}, \tau_{1}\right),\left(\pi_{2}, \tau_{2}\right), \ldots\right)$ of $\mathcal{S}$-valued random variables such that $\pi_{k}$ is $\mathcal{F}_{\tau_{k}}^{z}$-measurable and $\tau_{k}$ is a $\left(\mathcal{F}_{t}^{z}\right)$-stopping time, is an admissible trading strategy or an admissible portfolio for $z$. Thanks to the modified form of transaction costs no portfolio can lead to bankruptcy in a finite time. Let us denote the set of all admissible portfolios for $z$ by $\mathcal{A}^{z}$. For $z \in E$ and $\Pi \in \mathcal{A}^{z}$ we define the corresponding pre-transaction proportion process $\pi_{-}^{\Pi, z}(t)$ by

$$
\begin{aligned}
& \pi_{-}^{\Pi, z}(0)=\pi_{-}, \\
& \pi_{-}^{\Pi, z}(t)=\pi_{k} \diamond \zeta\left(\tau_{k}+1\right) \diamond \ldots \diamond \zeta(t), \quad \tau_{k}<t \leq \tau_{k+1},
\end{aligned}
$$

where for simplicity of the notation we set $\tau_{0}=0$ and

$$
\pi \diamond \zeta=g\left(\pi^{1} \zeta^{1}, \ldots, \pi^{d} \zeta^{d}\right), \quad \pi \in \mathcal{S}, \quad \zeta \in(0, \infty)^{d} .
$$

The corresponding post-transaction proportion process is given by

$$
\begin{aligned}
& \pi^{\Pi, z}(0)=\pi_{-}, \quad \tau_{1}>0, \\
& \pi^{\Pi, z}(t)=\pi_{k} \diamond \zeta\left(\tau_{k}+1\right) \diamond \ldots \diamond \zeta(t), \quad \tau_{k} \leq t<\tau_{k+1} .
\end{aligned}
$$

The wealth process $X_{-}^{\Pi, z}(t)$ is given by (11). In the sequel we shall skip the subscript $\Pi, z$ unless it leads to ambiguity. The goal of this paper is to maximize the functional

$$
J^{\pi_{-}, x_{-}, z}(\Pi)=\liminf _{T \rightarrow \infty} \frac{1}{T} \mathbb{E}^{z} \ln X_{-}(T)
$$


over all portfolios $\Pi \in \mathcal{A}^{z}$, where $\pi_{-}$is an initial proportion, $x_{-}$denotes initial wealth and $z$ is an initial state of the economic factor process. Observe that due to (11) we have

$$
\begin{aligned}
J^{\pi_{-}, x_{-}, z}(\Pi)=\liminf _{T \rightarrow \infty} \frac{1}{T}\{ & \sum_{t=0}^{T-1} \mathbb{E}^{z} \ln \pi(t) \cdot \zeta(t) \\
& \left.+\sum_{k=1}^{\infty} \mathbb{E}^{z}\left\{1_{\tau_{k}<T} \ln e\left(\pi_{-}\left(\tau_{k}\right), \pi_{k}, X_{-}\left(\tau_{k}\right)\right)\right\}\right\} .
\end{aligned}
$$

3. Existence of optimal strategies. Denote by $P(z, d y)$ the transition operator of the process $Z(t)$. We will need the following assumptions:

(A1) The process $(S(t), Z(t))$ satisfies the Feller property i.e. its transition operator maps the space of continuous bounded functions into itself.

(A2) $\mathcal{S} \times E \ni(\pi, z) \mapsto h(\pi, z)=\mathbb{E}^{z}\{\ln \pi \cdot \zeta(z(1), \xi(1))\}$ is a bounded, continuous function.

(A3) $\sup _{z, z^{\prime} \in E} \sup _{B \in \mathcal{E}}\left(P^{n}(z, B)-P^{n}\left(z^{\prime}, B\right)\right)=\kappa<1$ for some $n \geq 1$.

(A4) $\sup _{\pi_{-}, \pi \in \mathcal{S}} \sup _{z \in E} \mathbb{E}^{z} \frac{1}{e\left(\pi_{-}, \pi, x^{*}\right) \pi \cdot \zeta(z, \xi(1))}<1$.

We have

THEOREM 3.1. Under assumptions (A1)-(A4) there exists a measurable function $p: \mathcal{S} \times(0, \infty) \times E \rightarrow \mathcal{S}$, a constant $\lambda$ and a measurable set $I \subseteq \mathcal{S} \times(0, \infty) \times E$ such that

$$
\lambda=J^{\pi_{-}, x_{-}, z}\left(\Pi^{*}\right)=\sup _{\Pi \in \mathcal{A}} J^{\pi_{-}, x_{-}, z}(\Pi),
$$

where the optimal portfolio $\Pi^{*}=\left(\left(\pi_{1}^{*}, \tau_{1}^{*}\right),\left(\pi_{2}^{*}, \tau_{2}^{*}\right), \ldots\right)$ is given by the formulae

$$
\begin{aligned}
& \tau_{1}^{*}=\inf \left\{t \geq 0: \quad\left(\pi_{-}(t), X_{-}(t), Z(t)\right) \in I\right\}, \\
& \tau_{k+1}^{*}=\inf \left\{t>\tau_{k}^{*}: \quad\left(\pi_{-}(t), X_{-}(t), Z(t)\right) \in I\right\}, \\
& \pi_{k}^{*}=p\left(\pi_{-}\left(\tau_{k}^{*}\right), X_{-}\left(\tau_{k}^{*}\right), Z\left(\tau_{k}^{*}\right)\right) .
\end{aligned}
$$

Theorem 3.1 states that for any initial state of the market and for any initial share holding there exists an optimal portfolio maximizing the average growth rate. This portfolio has a Markovian structure: decision about a transaction at $t$ is based only on the state of the market at $t$ and not before $t$. This decision process is governed by the impulse set $I$ and the impulse function $p$. Clearly, every Markovian portfolio is admissible. Notice also that the optimal growth rate is equal to a constant $\lambda$ independently of the initial conditions. This is an inherent property of the so-called long-run average cost functionals (see [2], [9]). 


\section{Remarks.}

(1) Assume that $Z(t)$ is a Feller process, which is clearly required for (A1) to hold. If $\zeta^{i}(z, \xi), i=1, \ldots, d$, are continuous in $z$ then (A1) is satisfied. Indeed, let $\phi:(0, \infty)^{d} \times E \rightarrow \mathbb{R}$ be a continuous bounded function. Define

$$
g(s, z, \xi)=\int_{E} \phi\left(s^{1} \zeta^{1}(\tilde{z}, \xi), \ldots, s^{d} \zeta^{d}(\tilde{z}, \xi), \tilde{z}\right) P(z, d \tilde{z}) .
$$

It is continuous by the Feller property of $Z(t)$ and bounded by the boundedness of $\phi$. Consequently, the mapping

$$
(s, z) \mapsto \mathbb{E}^{(s, z)} \phi(S(1), Z(1))=\int_{E^{\xi}} g(s, z, \xi) \nu(d \xi),
$$

where $\nu$ is a distribution of $\xi(1)$ on $E^{\xi}$, is continuous by dominated convergence theorem and (A1) holds. In particular, if $Z(t)$ is a Markov chain with a finite state space (A1) is always satisfied.

(2) Notice that (A2) reads that expected one period growth rate is finite.

(3) Assume that $\zeta^{i}(z, \xi), i=1, \ldots, d$, are bounded functions separated from 0 and continuous in $z$. Consequently, $h(\pi, z)$ is bounded. By (A1) $Z(t)$ is a Feller process, hence $h(\pi, z)$ is continuous by the same argument as above and (A2) holds.

(4) By Jensen's inequality

$$
\inf _{\pi \in \mathcal{S}} h(z, \pi)=\min _{i=1, \ldots, d} \mathbb{E}^{z}\left\{\ln \zeta^{i}(Z(1), \xi(1))\right\} .
$$

Therefore, $h(\pi, z)$ is bounded from below if and only if

$$
\inf _{z \in E} \mathbb{E}^{z}\left\{\ln \zeta^{i}(Z(1), \xi(1))\right\}>-\infty, \quad i=1, \ldots, d .
$$

(5) Condition (A2) does not imply boundedness of $\zeta^{i}$. Consider a generalized Black-Scholes model with economic factors (see [3], [4], [13]), i.e.

$S^{i}(t+1)=S^{i}(t) \exp \left(\sigma^{i}(Z(t+1)) \cdot(W(t+1)-W(t))+\mu^{i}(Z(t+1))\right), \quad i=1, \ldots, d$,

where $Z(t)$ is a Feller process, $W(t)$ is an $m$-dimensional Wiener process and $\sigma^{i}$ : $E \rightarrow \mathbb{R}^{m}, \mu^{i}: E \rightarrow \mathbb{R}, i=1, \ldots, d$, are continuous bounded functions. Clearly, (A1) is satisfied by Remark (1). To show (A2) we recall the definition

$$
h(\pi, z)=\mathbb{E}^{z} \ln \left(\sum_{i=1}^{d} \pi^{i} \exp \left(\sigma^{i}(Z(1)) \cdot \xi(1)+\mu^{i}(Z(1))\right)\right)
$$

with $\xi(1)=W(1)-W(0)$. Consequently, $\mathbb{E}^{z}\left\{-D_{1}(Z(1))\|\xi(1)\|_{2}-D_{2}(Z(1))\right\} \leq h(\pi, z) \leq \mathbb{E}^{z}\left\{D_{1}(Z(1))\|\xi(1)\|_{2}+D_{2}(Z(1))\right\}$, 
where $\xi$ has a standard normal distribution $\nu$ on $\mathbb{R}^{m}, D_{1}(z)=\max _{i=1, \ldots, d}\left\|\sigma^{i}(z)\right\|_{2}$, $D_{2}(z)=\max _{i=1, \ldots, d}\left|\mu^{i}(z)\right|$ and $\|\cdot\|_{2}$ stands for the $L^{2}$ norm in $\mathbb{R}^{m}$. It proves boundedness of $h(\pi, z)$. Continuity with respect to $\pi$ follows by the dominated convergence theorem. By a similar argument

$$
\tilde{z} \mapsto \int_{\mathbb{R}^{m}} \ln \left(\sum_{i=1}^{d} \pi^{i} \exp \left(\sigma^{i}(\tilde{z}) \cdot \xi+\mu^{i}(\tilde{z})\right)\right) \nu(d \xi)
$$

is continuous. Hence, due to the Feller property of $Z(t)$, the function $h(\pi, z)$ is continuous with respect to $z$ and (A2) is satisfied. In particular, (A2) is satisfied if $Z(t)$ is a Markov chain with a finite state space.

(6) Assumption (A3) corresponds to uniform ergodicity of $Z(t)$ and implies, in particular, the existence of a unique invariant measure which is approximated uniformly by the iterations of the transition operator $P$ (see [7]).

(7) In the stochastic control literature a one-step uniform ergodicity is usually assumed, which is equivalent to (A3) with $n=1$ (see e.g. condition (UE) in [23]). Allowing for $n>1$ opened a new class of applications and is especially important in the financial context. It can be shown that (A3) is satisfied if $Z(t)$ is a recurrent Markov chain with a finite state space.

(8) Assumption (A4) links a transaction cost and a growth rate of one-stage investment. It says, in general, that no matter what strategy we choose the portfolio wealth is increasing on average.

4. Proof of Theorem 3.1. The proof uses a generalization of the vanishing discount method ([2], [9], [18], [23]) due to [18]. Main idea is to obtain a Bellman inequality for our optimization problem as a limit of modified Bellman equations for discounted problems related to (16). Given $\pi_{-}, x_{-}, z$ consider the functional

$J_{\beta}^{\pi_{-}, x_{-}, z}(\Pi)=\mathbb{E}^{z}\left\{\sum_{t=1}^{\infty} \beta^{t} h(\pi(t), Z(t))+\sum_{k=1}^{\infty} \beta^{\tau_{k}} \ln e\left(\pi_{-}\left(\tau_{k}\right), \pi_{k}, X_{-}\left(\tau_{k}\right)\right)\right\}, \beta \in(0,1)$,

and the value function

$$
v_{\beta}\left(\pi_{-}, x_{-}, z\right)=\sup _{\Pi \in \mathcal{A}^{z}} J_{\beta}^{\pi_{-}, x_{-}, z}(\Pi) .
$$

Denote by $M$ the impulse operator acting on measurable functions

$$
M w\left(\pi_{-}, x_{-}, z\right)=\sup _{\pi \in \mathcal{S}}\left\{\ln e\left(\pi_{-}, \pi, x_{-}\right)+w\left(\pi, x_{-} e\left(\pi_{-}, \pi, x_{-}\right), z\right)\right\} .
$$

LEMMA 4.1. The impulse operator maps the space of continuous bounded functions into itself. Moreover, given any bounded continuous function $w$ there exists a measurable selector for $M w$.

Proof. The proof is standard (see [9] or [17]). 
TheOREM 4.2. Under (A1)-(A2) the function $v_{\beta}$ is continuous and bounded, and satisfies the Bellman equation

$$
v_{\beta}\left(\pi_{-}, x_{-}, z\right)=\sup _{\tau} \mathbb{E}^{z}\left\{\sum_{t=0}^{\tau-1} \beta^{t} h(\pi(t), Z(t))+\beta^{\tau} M v_{\beta}\left(\pi_{-}(\tau), X_{-}(\tau), Z(\tau)\right)\right\},
$$

where $\pi_{-}(t)$ and $X_{-}(t)$ are processes representing the proportions and the wealth of the portfolio before transaction with the following dynamics: $\pi_{-}(0)=\pi_{-}, \pi_{-}(t+1)=$ $\pi_{-}(t) \diamond \zeta(t)$ and $X_{-}(0)=x_{-}, X_{-}(t+1)=X_{-}(t) \pi_{-}(t) \cdot \zeta(t)$.

Proof. By Lemma 2.1 the function $\ln e\left(\pi_{-}, \pi, x_{-}\right)$is bounded, and by (A2) $h(\pi, z)$ is bounded. Therefore, $v_{\beta}\left(\pi_{-}, x_{-}, z\right)$ is bounded. For a continuous bounded function $v: \mathcal{S} \times(0, \infty) \times E \mapsto \mathbb{R}$, let

$$
\mathcal{T}_{\beta} v(\pi, x, z)=\sup _{\tau} \mathbb{E}^{z}\left\{\sum_{t=0}^{\tau-1} \beta^{t} h(\pi(t), Z(t))+\beta^{\tau} M v\left(\pi_{-}(\tau), X_{-}(\tau), Z(\tau)\right)\right\} .
$$

Operator $\mathcal{T}_{\beta}$ maps the space $C^{b}=C^{b}(\mathcal{S} \times(0, \infty) \times E$; $\mathbb{R})$ of bounded continuous functions into itself. It follows from (A1), the Feller property of the transition operator of the process $(S(t), Z(t))$, by a general result on the continuity of the value function of optimal stopping problems. Let

$$
\left.v_{\beta}^{0}\left(\pi_{-}, x_{-}, z\right)=\sum_{t=0}^{\infty} \beta^{t} \mathbb{E}^{z} h\left(\pi_{-}(t)\right), X_{-}(t)\right), \quad v_{\beta}^{k+1}=\mathcal{T}_{\beta} v_{\beta}^{k} .
$$

Thanks to continuity of $v_{\beta}^{k}$ and $M v_{\beta}^{k}$ it can be shown that $v_{\beta}^{k}$ is a value function for the maximization of $J_{\beta}$ over admissible portfolios with at most $k$ transactions. Observe that it is never optimal to have two transactions at the same time $\left(\mathbb{P}\left(\tau_{k}=\tau_{k+1}\right)>0\right)$ due to subadditivity of the transaction cost structure. Therefore, we have the estimate

$$
\left\|v_{\beta}-v_{\beta}^{k}\right\|_{\infty} \leq \sum_{l=k}^{\infty} \beta^{l}\|h\|_{\infty}=\beta^{k} \frac{\|h\|_{\infty}}{1-\beta}
$$

which implies that $v_{\beta}^{k}$ tends uniformly to $v_{\beta}$. Consequently, $v_{\beta}$ is a continuous bounded function and satisfies $v_{\beta}=\mathcal{T}_{\beta} v_{\beta}$ equivalent to the Bellman equation (19).

4.1. Proportional transaction costs. Now we shall concentrate on the case without the constant term in the transaction cost function, i.e. when $c=0$. Consequently, $e\left(\pi_{-}, \pi, x_{-}\right)$satisfies the equation

$$
e\left(\pi_{-}, \pi, x_{-}\right)=1-c\left(\pi_{-}, e\left(\pi_{-}, \pi, x_{-}\right) \pi\right),
$$

and the function $e\left(\pi_{-}, \pi, x_{-}\right)$is independent of $x_{-}$, so we can denote it by $e\left(\pi_{-}, \pi\right)$. Since $J^{\pi_{-}, x_{-}, z}$ depends on the portfolio wealth only in the transaction costs term, which by the above comment no longer takes $X_{-}$into account, we can skip $x_{-}$

$$
J_{\beta}^{\pi_{-}, z}(\Pi)=\mathbb{E}^{z}\left\{\sum_{t=1}^{\infty} \beta^{t} h(\pi(t), Z(t))+\sum_{k=1}^{\infty} \beta^{\tau_{k}} \ln e\left(\pi_{-}\left(\tau_{k}\right), \pi_{k}\right)\right\}, \quad \beta \in(0,1) .
$$


Consequently, we shall denote by $v_{\beta}\left(\pi_{-}, z\right)$ the value function corresponding to this functional. By Theorem 4.2 it is bounded and continuous. However, in the sequel we shall need a boundedness property which is uniform in $\beta \in(0,1)$.

Lemma 4.3. For arbitrary $\beta \in(0,1), \pi_{-}, \pi_{-}^{\prime} \in \mathcal{S}, z \in E$

$$
v_{\beta}\left(\pi_{-}, z\right)-v_{\beta}\left(\pi_{-}^{\prime}, z\right) \leq-\ln \left(\inf _{\pi, \pi^{\prime}} e\left(\pi, \pi^{\prime}\right)\right)
$$

Proof. It is an easy consequence of the fact that for an arbitrary $\Pi=\left(\left(\pi_{1}, \tau_{1}\right),\left(\pi_{2}\right.\right.$, $\left.\left.\tau_{2}\right), \ldots\right) \in \mathcal{A}^{z}$

$$
J^{\pi_{-}, z}(\Pi) \leq J^{\pi_{-}^{\prime}, z}\left(\Pi^{\prime}\right)-\ln e\left(\pi_{-}^{\prime}, \pi_{-}\right),
$$

where $\Pi^{\prime}=\left(\left(\pi_{-}, 0\right),\left(\pi_{1}, \tau_{1}\right),\left(\pi_{2}, \tau_{2}\right), \ldots\right)$.

Lemma 4.4. Under (A3) there exists $M<\infty$ such that

$$
\left|v_{\beta}\left(\pi_{-}, z\right)-v_{\beta}\left(\pi_{-}^{\prime}, z^{\prime}\right)\right| \leq M,
$$

for $\beta \in(0,1), \pi_{-}, \pi_{-}^{\prime} \in \mathcal{S}, z, z^{\prime} \in E$.

Proof. Let $\underline{e}=\inf _{\pi_{-}, \pi \in \mathcal{S}} e\left(\pi_{-}, \pi\right)$. Fix $z, z^{\prime} \in E$ and $\pi_{-}, \pi_{-}^{\prime} \in \mathcal{S}$. Denote by $\Pi$ the portfolio optimal for $v_{\beta}\left(\pi_{-}, z\right)$, and by $\Pi^{\prime}$ the portfolio optimal for $v_{\beta}\left(\pi_{-}^{\prime}, z^{\prime}\right)$ (they exist due to Theorem 4.2). The corresponding proportion processes $\pi_{-}^{\Pi, z}(t), \pi_{-}^{\Pi^{\prime}, z^{\prime}}(t)$ will be written as $\pi_{-}(t), \pi_{-}^{\prime}(t)$ and the corresponding wealth processes $X_{-}^{\Pi, z}(t), X_{-}^{\Pi^{\prime}, z^{\prime}}(t)$ as $X_{-}(t), X_{-}^{\prime}(t)$. We have then

$$
\begin{aligned}
v_{\beta}\left(\pi_{-}, z\right)-v_{\beta}\left(\pi_{-}^{\prime}, z^{\prime}\right)= & \sum_{t=0}^{n-1} \beta^{t} \mathbb{E}^{z} h\left(\pi_{-}(t), z(t)\right)+\sum_{k=1}^{\infty} \mathbb{E}^{z}\left\{1_{\tau_{k}<n} \beta^{\tau_{k}} \ln e\left(\pi_{-}\left(\tau_{k}\right), \pi_{k}\right)\right\} \\
& -\sum_{t=0}^{n-1} \beta^{t} \mathbb{E}^{z^{\prime}} h\left(\pi_{-}^{\prime}(t), z^{\prime}(t)\right) \\
& -\sum_{k=1}^{\infty} \mathbb{E}^{z^{\prime}}\left\{1_{\tau_{k}<n} \beta^{\tau_{k}} \ln e\left(\pi_{-}^{\prime}\left(\tau_{k}\right), \pi_{k}\right)\right\} \\
& +\beta^{n}\left(\mathbb{E}^{z} v_{\beta}\left(\pi_{-}(n), z(n)\right)-\mathbb{E}^{z^{\prime}} v_{\beta}\left(\pi_{-}^{\prime}(n), z^{\prime}(n)\right)\right) .
\end{aligned}
$$

There are at most $n$ transactions between 0 and $n-1$, since it is never optimal to have more than one transaction at a moment (by subadditivity of the cost function). Due to the fact that $h$ is bounded and $-\infty<\ln \underline{e} \leq \ln e\left(\pi_{-}, \pi\right) \leq 0$ by Lemma 2.1, we have

$$
\begin{aligned}
& v_{\beta}\left(\pi_{-}, z\right)-v_{\beta}\left(\pi_{-}^{\prime}, z^{\prime}\right) \\
\leq & n\|h\|_{s p}-n \ln \underline{e}+\beta^{n}\left(\mathbb{E}^{z} v_{\beta}\left(\pi_{-}(n), z(n)\right)-\mathbb{E}^{z^{\prime}} v_{\beta}\left(\pi_{-}^{\prime}(n), z^{\prime}(n)\right)\right),
\end{aligned}
$$


where $\|f\|_{s p}=\sup f-\inf f$ is the span semi-norm. Choose arbitrary $\pi^{*} \in \mathcal{S}$ and observe that

$$
\begin{aligned}
& \mathbb{E}^{z} v_{\beta}\left(\pi_{-}(n), z(n)\right)-\mathbb{E}^{z^{\prime}} v_{\beta}\left(\pi_{-}^{\prime}(n), z^{\prime}(n)\right) \\
= & \mathbb{E}^{z}\left\{v_{\beta}\left(\pi_{-}(n), z(n)\right)-v_{\beta}\left(\pi^{*}, z(n)\right)\right\}+\mathbb{E}^{z^{\prime}}\left\{v_{\beta}\left(\pi^{*}, z^{\prime}(n)\right)-v_{\beta}\left(\pi_{-}^{\prime}(n), z^{\prime}(n)\right)\right\} \\
& +\mathbb{E}^{z} v_{\beta}\left(\pi^{*}, z(n)\right)-\mathbb{E}^{z^{\prime}} v_{\beta}\left(\pi^{*}, z^{\prime}(n)\right) .
\end{aligned}
$$

By Lemma 4.3 we have

$$
\begin{aligned}
\mathbb{E}^{z}\left\{v_{\beta}\left(\pi_{-}(n), z(n)\right)-v_{\beta}\left(\pi^{*}, z(n)\right)\right\} & \leq-\ln \underline{\underline{e}}, \\
\mathbb{E}^{z^{\prime}}\left\{v_{\beta}\left(\pi^{*}, z^{\prime}(n)\right)-v_{\beta}\left(\pi_{-}^{\prime}(n), z^{\prime}(n)\right)\right\} & \leq-\ln \underline{e} .
\end{aligned}
$$

Notice that

$$
\begin{aligned}
& \mathbb{E}^{z} v_{\beta}\left(\pi^{*}, z(n)\right)-\mathbb{E}^{z^{\prime}} v_{\beta}\left(\pi^{*}, z^{\prime}(n)\right) \\
= & \int_{E} v_{\beta}\left(\pi^{*}, y\right) d P^{n}(z, d y)-\int_{E} v_{\beta}\left(\pi^{*}, y\right) d P^{n}\left(z^{\prime}, d y\right) \\
= & \int_{E} v_{\beta}\left(\pi^{*}, y\right) q(d y),
\end{aligned}
$$

with $q=P^{n}(z, \cdot)-P^{n}\left(z^{\prime}, \cdot\right)$. Let $\Gamma \in \mathcal{E}$ be the set coming from the Hahn-Jordan decomposition of the signed measure $q$, i.e. $q$ is non-negative on $\Gamma$ and non-positive on $\Gamma^{c}$. By $(\mathrm{A} 3)$

$$
\begin{aligned}
\int_{E} v_{\beta}\left(\pi^{*}, y\right) q(d y)= & \int_{E}\left(v_{\beta}\left(\pi^{*}, y\right)-\inf _{y^{\prime} \in E} v_{\beta}\left(\pi^{*}, y^{\prime}\right)\right) q(d y) \\
\leq & \int_{\Gamma}\left(v_{\beta}\left(\pi^{*}, y\right)-\inf _{y^{\prime} \in E} v_{\beta}\left(\pi^{*}, y^{\prime}\right)\right) q(d y) \\
& +\int_{\Gamma^{c}}\left(v_{\beta}\left(\pi^{*}, y\right)-\inf _{y^{\prime} \in E} v_{\beta}\left(\pi^{*}, y^{\prime}\right)\right) q(d y) \\
\leq & \left\|v_{\beta}\left(\pi^{*}, \cdot\right)\right\|_{s p} q(\Gamma) \leq \kappa\left\|v_{\beta}\left(\pi^{*}, \cdot\right)\right\|_{s p} .
\end{aligned}
$$

Consequently,

$$
v_{\beta}\left(\pi_{-}, z\right)-v_{\beta}\left(\pi_{-}^{\prime}, z^{\prime}\right) \leq n\|h\|_{s p}-(n+2) \ln \underline{e}+\kappa\left\|v_{\beta}\left(\pi^{*}, \cdot\right)\right\|_{s p} .
$$

Since $\pi_{-}, \pi_{-}^{\prime} \in \mathcal{S}$ and $z, z^{\prime} \in E$ were arbitrary we obtain

$$
\left\|v_{\beta}\left(\pi^{*}, \cdot\right)\right\|_{s p} \leq n\|h\|_{s p}-(n+2) \ln \underline{e}+\kappa\left\|v_{\beta}\left(\pi^{*}, \cdot\right)\right\|_{s p},
$$

which yields the required result with

$$
M=\frac{n\|h\|_{s p}-(n+2) \ln \underline{e}}{1-\kappa} .
$$


4.2. Technical estimates. We shall derive the estimates on the diminution of the portfolio wealth under transaction costs.

Lemma 4.5. For $\pi_{-}, \pi \in \mathcal{S}, x_{-} \in(0, \infty)$

i) $e\left(\pi_{-}, \pi\right) \geq e\left(\pi_{-}, \pi, x_{-}\right) \geq e\left(\pi_{-}, \pi, \tilde{x}_{-}\right), \quad x_{-} \geq \tilde{x}_{-}>0$.

ii) $e\left(\pi_{-}, \pi\right)-e\left(\pi_{-}, \pi, x_{-}\right) \leq \frac{c}{\left(x_{-} \vee x^{*}\right)\left(1-\max _{i} c_{i}^{1}\right)}$.

iii) $\ln \frac{e\left(\pi_{-}, \pi\right)}{e\left(\pi_{-}, \pi, x_{-}\right)} \leq \frac{1}{\inf _{\tilde{\pi}_{-}, \tilde{\pi}} e\left(\tilde{\pi}_{-}, \tilde{\pi}, x^{*}\right)} \frac{c}{\left(x_{-} \vee x^{*}\right)\left(1-\max _{i} c_{i}^{1}\right)}$.

Proof. Noticing $a^{+}-b^{+} \leq(a-b)^{+}$and $a^{-}-b^{-} \leq(a-b)^{-}$we obtain for $\delta_{1}, \delta_{2} \in[0,1]$

$$
\begin{aligned}
c\left(\pi_{-}, \delta_{2} \pi\right)-c\left(\pi_{-}, \delta_{1} \pi\right)= & \sum_{i=1}^{d}\left(c_{i}^{1}\left(\left(\pi_{-}\right)_{i}-\delta_{2} \pi_{i}\right)^{+}-c_{i}^{1}\left(\left(\pi_{-}\right)_{i}-\delta_{1} \pi_{i}\right)^{+}\right. \\
& \left.+c_{i}^{2}\left(\left(\pi_{-}\right)_{i}-\delta_{2} \pi_{i}\right)^{-}-c_{i}^{2}\left(\left(\pi_{-}\right)_{i}-\delta_{1} \pi_{i}\right)^{-}\right) \\
\leq & \sum_{i=1}^{d}\left(c_{i}^{1}\left(\delta_{1}-\delta_{2}\right)^{+} \pi_{i}+c_{i}^{2}\left(\delta_{1}-\delta_{2}\right)^{-} \pi_{i}\right) .
\end{aligned}
$$

Consequently,

$$
\left|c\left(\pi_{-}, \delta_{2} \pi\right)-c\left(\pi_{-}, \delta_{1} \pi\right)\right| \leq\left|\delta_{2}-\delta_{1}\right| \max _{i}\left(c_{i}^{1}, c_{i}^{2}\right) .
$$

By definition we have

$$
\begin{aligned}
& e\left(\pi_{-}, \pi\right)=1-c\left(\pi_{-}, e\left(\pi_{-}, \pi\right) \pi\right), \\
& e\left(\pi_{-}, \pi, x_{-}\right)=1-c\left(\pi_{-}, e\left(\pi_{-}, \pi, x_{-}\right) \pi\right)-\frac{c}{x_{-} \vee x^{*}} .
\end{aligned}
$$

We shall prove (i) by contradiction. Assume that $e\left(\pi_{-}, \pi\right)<e\left(\pi_{-}, \pi, x_{-}\right)$. Easily,

$$
0 \leq e\left(\pi_{-}, \pi, x_{-}\right)-e\left(\pi_{-}, \pi\right) \leq c\left(\pi_{-}, e\left(\pi_{-}, \pi\right) \pi\right)-c\left(\pi_{-}, e\left(\pi_{-}, \pi, x_{-}\right) \pi\right)-\frac{c}{x_{-} \vee x^{*}} .
$$

By (21) we obtain

$$
e\left(\pi_{-}, \pi, x_{-}\right)-e\left(\pi_{-}, \pi\right) \leq\left(e\left(\pi_{-}, \pi, x_{-}\right)-e\left(\pi_{-}, \pi\right)\right) \max _{i}\left(c_{i}^{1}, c_{i}^{2}\right)-\frac{c}{x_{-} \vee x^{*}} .
$$

It gives the estimate

$$
1+\frac{c}{x_{-} \vee x^{*}\left(e\left(\pi_{-}, \pi, x_{-}\right)-e\left(\pi_{-}, \pi\right)\right)} \leq \max _{i}\left(c_{i}^{1}, c_{i}^{2}\right),
$$

which contradicts the assumption that $c_{i}^{1}, c_{i}^{2} \in[0,1)$. The proof of $e\left(\pi_{-}, \pi, x_{-}\right) \leq$ $e\left(\pi_{-}, \pi, \tilde{x}_{-}\right)$follows a similar line of argument.

Notice that from (i), (22) and (20) we obtain

$$
\begin{aligned}
e\left(\pi_{-}, \pi\right)-e\left(\pi_{-}, \pi, x_{-}\right) & =c\left(\pi_{-}, e\left(\pi_{-}, \pi, x_{-}\right) \pi\right)-c\left(\pi_{-}, e\left(\pi_{-}, \pi\right) \pi\right)+\frac{c}{x_{-} \vee x^{*}} \\
& \leq\left(e\left(\pi_{-}, \pi\right)-e\left(\pi_{-}, \pi, x_{-}\right)\right) \max _{i} c_{i}^{1}+\frac{c}{x_{-} \vee x^{*}},
\end{aligned}
$$


which immediately proves (ii). For (iii) we use the inequality $\ln (1+x) \leq x$ for $x>0$.

Corollary 4.6. The value function $v_{\beta}\left(\pi_{-}, x_{-}, z\right)$ is non-decreasing in $x_{-}$.

Proof. Given $\pi_{-} \in \mathcal{S}, z \in E$ and $\tilde{x}_{-} \leq x_{-}$

$$
v_{\beta}\left(\pi_{-}, \tilde{x}_{-}, z\right)-v_{\beta}\left(\pi_{-}, x_{-}, z\right) \leq \sup _{\Pi \in \mathcal{A}^{z}}\left\{J_{\beta}^{\pi, \tilde{x}_{-}, z}(\Pi)-J_{\beta}^{\pi, x_{-}, z}(\Pi)\right\} .
$$

Fix $\pi \in \mathcal{A}^{z}$ and observe that

$$
\begin{aligned}
& J_{\beta}^{\pi_{-}, \tilde{x}_{-}, z}(\Pi)-J_{\beta}^{\pi_{-}, x_{-}, z}(\Pi) \\
= & \sum_{k=1}^{\infty} \beta^{\tau_{k}}\left(\ln e\left(\pi_{-}\left(\tau_{k}\right), \pi_{k}, \tilde{X}_{-}\left(\tau_{k}\right)\right)-\ln e\left(\pi_{-}\left(\tau_{k}\right), \pi_{k}, X_{-}\left(\tau_{k}\right)\right)\right),
\end{aligned}
$$

where $\tau_{0}=0, \pi_{-}(0)=\pi_{-}$,

$$
\pi_{-}(t)=\pi_{k} \diamond \zeta\left(\tau_{k}+1\right) \diamond \ldots \diamond \zeta(t), \quad \tau_{k}<t \leq \tau_{k+1}
$$

and $X_{-}(t), \tilde{X}_{-}(t)$ are given by (11). By Lemma 4.5 (i) we have $X_{-}(t) \geq \tilde{X}_{-}(t), t \geq 0$ and consequently $J_{\beta}^{\pi, \tilde{x}_{-}, z}(\Pi)-J_{\beta}^{\pi, x_{-}, z}(\Pi) \leq 0$.

Lemma 4.7. Under (A4), there exists a constant $D>0$ such that for $\pi_{-} \in \mathcal{S}$, $z \in E, x_{-}>0$

$$
0 \leq v_{\beta}\left(\pi_{-}, z\right)-v_{\beta}\left(\pi_{-}, x_{-}, z\right) \leq \frac{D}{x_{-}}, \quad \beta \in(0,1) .
$$

Proof. It suffices to obtain an estimate for

$$
J_{\beta}^{\pi_{-}, z}(\Pi)-J_{\beta}^{\pi_{-}, x_{-}, z}(\Pi)
$$

independent of $z \in E, \Pi \in \mathcal{A}^{z}, \pi_{-} \in \mathcal{S}$, and $\beta \in(0,1)$. Since

$$
J_{\beta}^{\pi_{-}, z}(\Pi)-J_{\beta}^{\pi_{-}, x_{-}, z}(\Pi)=\sum_{k=1}^{\infty} \mathbb{E}^{z}\left\{\beta^{\tau_{k}} \ln \frac{e\left(\pi_{-}\left(\tau_{k}\right), \pi_{k}\right)}{e\left(\pi_{-}\left(\tau_{k}\right), \pi_{k}, X_{-}\left(\tau_{k}\right)\right)}\right\},
$$

Lemma 4.5 (i) implies that $J_{\beta}^{\pi_{-}, z}(\Pi)-J_{\beta}^{\pi_{-}, x_{-}, z}(\Pi) \geq 0$. To obtain the second inequality notice that as in the proof of Theorem 4.2 we can restrict ourselves to portfolios with at most one transaction at a moment. By Lemma 4.5

$$
\begin{aligned}
J_{\beta}^{\pi_{-}, z}(\Pi)-J_{\beta}^{\pi_{-}, x_{-}, z}(\Pi) & =\sum_{k=1}^{\infty} \mathbb{E}^{z}\left\{\beta^{\tau_{k}} \ln \frac{e\left(\pi_{-}\left(\tau_{k}\right), \pi_{k}\right)}{e\left(\pi_{-}\left(\tau_{k}\right), \pi_{k}, X_{-}\left(\tau_{k}\right)\right)}\right\} \\
& \leq \sum_{k=1}^{\infty} \mathbb{E}^{z} \frac{d}{x^{*} \vee X_{-}\left(\tau_{k}\right)} \leq \sum_{t=0}^{\infty} \mathbb{E}^{z} \frac{d}{X_{-}(t)},
\end{aligned}
$$

where

$$
d=\frac{1}{\inf _{\tilde{\pi}_{-}, \tilde{\pi}} e\left(\tilde{\pi}_{-}, \tilde{\pi}, x^{*}\right)} \frac{c}{\left(1-\max _{i} c_{i}^{1}\right)} .
$$


Let

$$
\Delta=\sup _{\pi_{-}, \pi \in \mathcal{S}} \sup _{z \in E} \mathbb{E}^{z} \frac{1}{e\left(\pi_{-}, \pi, x^{*}\right) \pi \cdot \zeta(z, \xi(1))}
$$

and notice that $\Delta<1$ by (A4). Applying Jensen's inequality and the following estimate

$$
X_{-}(t) \geq x_{-} \prod_{s=0}^{t-1} e\left(\pi_{-}(s), \pi(s), X_{-}(s)\right) \pi(s) \cdot \zeta(s+1)
$$

we obtain

$$
\sum_{t=0}^{\infty} \mathbb{E}^{z} \frac{d}{X_{-}(t)} \leq \frac{d}{(1-\Delta) x_{-}}
$$

4.3. Bellman inequality. Denote by $\mathcal{H}=\mathcal{S} \times(0, \infty) \times E$ the state space of our Markovian control model. It is locally compact, which will be needed in Lemma 4.9. Denote by $q$ a controlled transition operator, i.e. a function $q: \mathcal{H} \times \mathcal{S} \rightarrow \mathcal{P}(\mathcal{H})$, where $\mathcal{P}(\mathcal{H})$ is the space of Borel probability measures on $\mathcal{H}$, given by the following relation: for any bounded measurable function $f: \mathcal{H} \rightarrow \mathbb{R}$

$$
\int_{\mathcal{H}} f\left(\tilde{\pi}_{-}, \tilde{x}_{-}, \tilde{z}\right) q\left(\pi_{-}, x_{-}, z, \pi\right)\left(d \tilde{\pi}_{-}, d \tilde{x}_{-}, d \tilde{z}\right)=\mathbb{E}^{z} f\left(\pi \diamond \zeta(z, \xi(1)), X_{-}(1), z(1)\right),
$$

where

$$
X_{-}(1)= \begin{cases}x_{-} e\left(\pi_{-}, \pi, x_{-}\right)(\pi \cdot \zeta(z, \xi(1))), & \text { when } \pi_{-} \neq \pi, \\ x_{-} \pi \cdot \zeta(z, \xi(1)), & \text { when } \pi_{-}=\pi\end{cases}
$$

One can see that $q$ is not continuous in any reasonable sense as long as the constant term in transaction costs is non-null. Indeed, $x_{-} \pi \cdot \zeta(z, \xi(1))-x_{-} e\left(\pi_{-}, \pi, x_{-}\right)(\pi$. $\zeta(z, \xi(1))) \geq c$.

We cannot apply results known for the vanishing discount approach, since they require continuity of the transition operator $q$ and a uniform bound on the span seminorm of $v_{\beta}$ (see [18], [9], [10]). Instead, we shall modify the approach of [18] making use of the estimates derived in the previous sections.

To simplify the notation consider

$$
\eta\left(\pi_{-}, \pi, x_{-}, z\right)= \begin{cases}h(\pi, z), & \pi_{-}=\pi, \\ h(\pi, z)+\ln e\left(\pi_{-}, \pi, x_{-}\right), & \pi_{-} \neq \pi .\end{cases}
$$

The Bellman equation (19) has an equivalent form

$$
v_{\beta}\left(\pi_{-}, x_{-}, z\right)=\sup _{\pi \in \mathcal{S}}\left\{\eta\left(\pi_{-}, \pi, x_{-}, z\right)+\beta \int v_{\beta} d q\left(\pi_{-}, x_{-}, z, \pi\right)\right\} .
$$


Let $a_{\beta}: \mathcal{H} \rightarrow \mathcal{S}$ be a measurable selector for $M v_{\beta}$ (see (18)) and $I_{\beta}$ be the impulse region

$$
I_{\beta}=\left\{\left(\pi_{-}, x_{-}, z\right) \in \mathcal{H}: \quad v_{\beta}\left(\pi_{-}, x_{-}, z\right)=M v_{\beta}\left(\pi_{-}, x_{-}, z\right)\right\}
$$

The optimal strategy in this formulation is given by a measurable function $f_{\beta}: \mathcal{H} \rightarrow \mathcal{S}$

$$
f_{\beta}\left(\pi_{-}, x_{-}, z\right)= \begin{cases}\pi_{-}, & \left(\pi_{-}, x_{-}, z\right) \notin I_{\beta}, \\ a_{\beta}\left(\pi_{-}, x_{-}, z\right), & \left(\pi_{-}, x_{-}, z\right) \in I_{\beta} .\end{cases}
$$

Since $v_{\beta}$ is not uniformly bounded in $\beta$ we introduce the relative discounted value function

$$
w_{\beta}\left(\pi_{-}, x_{-}, z\right)=m_{\beta}-v_{\beta}\left(\pi_{-}, x_{-}, z\right)
$$

where

$$
m_{\beta}=\sup _{\pi_{-} \in \mathcal{S}} \sup _{z \in E} v_{\beta}\left(\pi_{-}, z\right) .
$$

We have

LEMMA 4.8.

i) $0 \leq w_{\beta}\left(\pi_{-}, x_{-}, z\right) \leq M+\frac{D}{x_{-}}$with $M, D>0$ independent of $\beta, \pi_{-}, x_{-}, z$.

ii) The set $\left\{(1-\beta) m_{\beta}: \beta \in(0,1)\right\}$ is compact.

Proof. By Lemma 4.4 and 4.7

$$
w_{\beta}\left(\pi_{-}, x_{-}, z\right) \leq m_{\beta}-v_{\beta}\left(\pi_{-}, z\right)+v_{\beta}\left(\pi_{-}, z\right)-v_{\beta}\left(\pi_{-}, x_{-}, z\right) \leq M+\frac{D}{x_{-}} .
$$

Part (ii) follows from boundedness of $\eta$.

Let $\bar{\lambda}=\lim \sup _{\beta \uparrow 1} m_{\beta}$, which is finite by statement (ii) of Lemma 4.8. Denote by $\beta_{k}$ the sequence of discount factors converging to 1 such that

$$
\bar{\lambda}=\lim _{k \rightarrow \infty} m_{\beta_{k}} .
$$

Write

$$
w(k, \vartheta)=w_{\beta_{k}}(\vartheta), \quad \underline{w}(\vartheta)=\liminf _{k \rightarrow \infty, \vartheta^{\prime} \rightarrow \vartheta} w\left(k, \vartheta^{\prime}\right), \quad \vartheta \in \mathcal{H} .
$$

Lemma 4.9. ([18] Lemma 3.4) Assume that $\mathcal{H}$ is locally compact. There exist sequences of measurable mappings $\left\{k_{n}\right\}, k_{n}: \mathcal{H} \rightarrow \mathbb{N}$ and $\left\{\theta_{n}\right\}, \theta_{n}: \mathcal{H} \rightarrow \mathcal{H}$ such that

i) $k_{n}(\vartheta) \rightarrow \infty, \theta_{n}(\vartheta) \rightarrow \vartheta$ as $n \rightarrow \infty$ for any $\vartheta \in \mathcal{H}$,

ii) $w\left(k_{n}(\vartheta), \theta_{n}(\vartheta)\right) \rightarrow \underline{w}(\vartheta)$ as $n \rightarrow \infty$.

In particular, $\underline{w}$ is measurable. 
In the sequel we shall need two transition operators related to $q$. Let $\underline{q}$ be given by the formula (23) with

$$
X_{-}(1)=x_{-} e\left(\pi_{-}, \pi, x_{-}\right)(\pi \cdot \zeta(z, \xi(1))),
$$

and $\bar{q}$ with

$$
X_{-}(1)=x_{-}(\pi \cdot \zeta(z, \xi(1))) \text {. }
$$

They are weakly continuous. Indeed, it is straightforward by (A1) and the continuity of $e\left(\pi_{-}, \pi, x_{-}\right)$(see Lemma 2.1) that

$$
\left(\pi_{-}, x_{-}, z\right) \mapsto\left(\int_{\mathcal{H}} f d \underline{q}\left(\pi_{-}, x_{-}, z\right), \int_{\mathcal{H}} f d \bar{q}\left(\pi_{-}, x_{-}, z\right)\right)
$$

is continuous for any continuous bounded function $f: \mathcal{H} \rightarrow \mathbb{R}$.

Lemma 4.10. ([16] Lemma 3.2) Let $\left\{\mu_{n}\right\}$ be a sequence of probability measures on a separable metric space $\mathcal{X}$ converging weakly to $\mu$ and $\left\{g_{n}\right\}$ be a sequence of measurable nonnegative functions on $\mathcal{X}$. Then

$$
\int \underline{g} d \mu \leq \liminf _{n \rightarrow \infty} \int g_{n} d \mu_{n}, \quad \text { where } \quad \underline{g}(x)=\liminf _{n \rightarrow \infty, y \rightarrow x} g_{n}(y), \quad x \in \mathcal{X} .
$$

Now we are ready to derive a Bellman inequality which is the main constitute of the proof of Theorem 3.1.

THEOREM 4.11. Under assumptions (A1)-(A4) there exists a measurable function $f_{1}: \mathcal{H} \rightarrow \mathcal{S}$ and a measurable function $w: \mathcal{H} \rightarrow(-\infty, 0]$ such that

$$
w(\vartheta)+\bar{\lambda} \leq \eta\left(\vartheta, f_{1}(\vartheta)\right)+\int w\left(\vartheta^{\prime}\right) q\left(\vartheta, f_{1}(\vartheta)\right)\left(d \vartheta^{\prime}\right), \quad \vartheta \in \mathcal{H} .
$$

Proof. From equation (24) we obtain

$$
w_{\beta}(\vartheta)+(\beta-1) m_{\beta}=-\eta\left(\vartheta, f_{\beta}(\vartheta)\right)+\beta \int w_{\beta}\left(\vartheta^{\prime}\right) q\left(\vartheta, f_{\beta}(\vartheta)\right)\left(d \vartheta^{\prime}\right), \vartheta \in \mathcal{H}, \beta \in(0,1),
$$

where $f_{\beta}$ is the optimal strategy for $v_{\beta}$. In the notation of Lemma 4.9

$$
\begin{aligned}
w\left(k_{n}(\vartheta), \theta_{n}(\vartheta)\right) & +(\beta(n, \vartheta)-1) m_{\beta(n, \vartheta)} \\
& =-\eta\left(\theta_{n}(\vartheta), s_{n}(\vartheta)\right)+\beta(n, \vartheta) \int w\left(k_{n}(\vartheta), \vartheta^{\prime}\right) q\left(\theta_{n}(\vartheta), s_{n}(\vartheta)\right)\left(d \vartheta^{\prime}\right),
\end{aligned}
$$

where

$$
\beta(n, \vartheta)=\beta_{k_{n}(\vartheta)}, \quad s_{n}(\vartheta)=f_{\beta(n, \vartheta)}\left(\theta_{n}(\vartheta)\right)
$$

Since $\mathcal{S}$ is compact the set of accumulation points of $\left\{s_{n}(\vartheta)\right\}_{n=1,2, \ldots}$ is non-empty. Following [17] Lemma 4 we can find a measurable selector of accumulation points 
i.e. a measurable function $f_{1}: \mathcal{H} \rightarrow \mathcal{S}$ such that $f_{1}(\vartheta)$ is an accumulation point of $\left\{s_{n}(\vartheta)\right\}_{n=1,2, \ldots}$. Fix $\vartheta \in \mathcal{H}$. There exists a subsequence $\left(n_{k}\right)$ such that $s_{n_{k}}(\vartheta) \rightarrow f_{1}(\vartheta)$ and either (a) $\theta_{n_{k}}(\vartheta) \in I_{\beta\left(n_{k}, \vartheta\right)}$ for every $k$, or (b) $\theta_{n_{k}}(\vartheta) \notin I_{\beta\left(n_{k}, \vartheta\right)}$ for every $k$. Assume first that (a) holds. From

$$
\int w\left(k_{n}(\vartheta), \vartheta^{\prime}\right) q\left(\theta_{n}(\vartheta), s_{n}(\vartheta)\right)\left(d \vartheta^{\prime}\right)=\int w\left(k_{n}(\vartheta), \vartheta^{\prime}\right) \underline{q}\left(\theta_{n}(\vartheta), s_{n}(\vartheta)\right)\left(d \vartheta^{\prime}\right)
$$

and Lemma 4.10 we obtain

$$
\liminf _{n \rightarrow \infty} \int w\left(k_{n}(\vartheta), \vartheta^{\prime}\right) q\left(\theta_{n}(\vartheta), s_{n}(\vartheta)\right)\left(d \vartheta^{\prime}\right) \geq \int \underline{w}\left(\vartheta^{\prime}\right) \underline{q}\left(\vartheta, f_{1}(\vartheta)\right)\left(d \vartheta^{\prime}\right) .
$$

By Corollary 4.6 the functions $v_{\beta}\left(\pi_{-}, x_{-}, z\right)$ are non-decreasing in $x_{-}$. This implies that $\underline{w}\left(\pi_{-}, x_{-}, z\right)$ is non-increasing in $x_{-}$. Hence $\int \underline{w}\left(\vartheta^{\prime}\right) \underline{q}\left(\vartheta, f_{1}(\vartheta)\right)\left(d \vartheta^{\prime}\right) \geq$ $\int \underline{w}\left(\vartheta^{\prime}\right) q\left(\vartheta, f_{1}(\vartheta)\right)\left(d \vartheta^{\prime}\right)$ and

$$
\liminf _{n \rightarrow \infty} \int w\left(k_{n}(\vartheta), \vartheta^{\prime}\right) q\left(\theta_{n}(\vartheta), s_{n}(\vartheta)\right)\left(d \vartheta^{\prime}\right) \geq \int \underline{w}\left(\vartheta^{\prime}\right) q\left(\vartheta, f_{1}(\vartheta)\right)\left(d \vartheta^{\prime}\right) .
$$

In case (b) we have $s_{n_{k}}(\vartheta)=\pi_{-}^{n}$, where $\theta_{n}(\vartheta)=\left(\pi_{-}^{n}, x_{-}^{n}, z^{n}\right)$. Since $\theta_{n}(\vartheta) \rightarrow \vartheta$ and $s_{n_{k}}(\vartheta) \rightarrow f_{1}(\vartheta)$ we have $f_{1}(\vartheta)=\pi_{-}$, where $\vartheta=\left(\pi_{-}, x_{-}, z\right)$. From equalities $q\left(\theta_{n}(\vartheta), s_{n}(\vartheta)\right)=\bar{q}\left(\theta_{n}(\vartheta), s_{n}(\vartheta)\right)$ and $q\left(\vartheta, f_{1}(\vartheta)\right)=\bar{q}\left(\vartheta, f_{1}(\vartheta)\right)$ and Lemma 4.10 we obtain (27). Since $\eta$ is upper semicontinuous we conclude from (26) that

$$
\underline{w}(\vartheta)-\bar{\lambda} \geq-\eta\left(\vartheta, f_{1}(\vartheta)\right)+\int \underline{w}\left(\vartheta^{\prime}\right) q\left(\vartheta, f_{1}(\vartheta)\right)\left(d \vartheta^{\prime}\right),
$$

which yields $(25)$ with $w=-\underline{w}$.

Proof of Theorem 3.1. Fix $\left(\pi_{-}, x_{-}, z\right) \in \mathcal{H}$ and define a portfolio $\Pi=\left(\left(\pi_{1}, \tau_{1}\right),\left(\pi_{2}\right.\right.$, $\left.\left.\tau_{2}\right), \ldots\right)$ by formulae given in Theorem 3.1 with $I=\left\{\left(\pi_{-}, x_{-}, z\right) \in \mathcal{H}: f_{1}\left(\pi_{-}, x_{-}, z\right) \neq\right.$ $\left.\pi_{-}\right\}$and $p=f_{1}$. Iterating (25) $T$ times, dividing by $T$ and passing with $T$ to infinity we obtain

$$
\bar{\lambda} \leq J^{\pi_{-}, x_{-}, z}(\Pi)+\liminf _{T \rightarrow \infty} \mathbb{E}^{z} \frac{w\left(\pi_{-}^{\Pi}(T), X_{-}^{\Pi}(T), Z(T)\right)}{T} \leq J^{\pi_{-}, x_{-}, z}(\Pi),
$$

since $w$ is nonpositive. On the other hand, by a well-known Tauberian relation

$$
\begin{aligned}
J^{\pi_{-}, x_{-}, z}(\Pi) & \leq \liminf _{\beta \rightarrow 1}(1-\beta) J_{\beta}^{\pi_{-}, x_{-}, z}(\Pi) \\
& \leq \liminf _{\beta \rightarrow 1}(1-\beta) v_{\beta}\left(\pi_{-}, x_{-}, z\right) \leq \liminf _{\beta \rightarrow 1}(1-\beta) v_{\beta}\left(\pi_{-}, z\right) \leq \bar{\lambda},
\end{aligned}
$$

which proves the optimality of $\Pi$.

COROLlary 4.12. The portfolio constructed above is optimal for the case with the term $c$ of the transaction cost function equal to 0 . It yields the optimal average growth rate equal to $\bar{\lambda}$. 
Proof. First notice that $\bar{\lambda}$ is the optimal value for the problem with proportional transaction costs. Indeed, consider the proof of Theorem 4.11 with $w_{\beta}\left(\pi_{-}, z\right)=$ $m_{\beta}-v_{\beta}\left(\pi_{-}, z\right)$. We obtain an analog of (25) with function $w$ depending on $\pi_{-}, z$ and $\bar{\lambda}$ as above. Consequently $\bar{\lambda}$ is the optimal value for the problem with proportional transaction costs.

Let $\Pi$ be the optimal portfolio for the case with fixed and proportional transaction costs (as defined in Theorem 3.1). Denote by $\bar{X}^{\Pi}(t)$ the wealth of the portfolio governed by $\Pi$ when the fixed term of the transaction cost function is equal to 0 . Obviously $\bar{X}^{\Pi}(t) \geq X^{\Pi}(t)$ and

$$
\lim _{T \rightarrow \infty} \frac{1}{T} \mathbb{E}^{z} \ln \bar{X}^{\Pi}(t) \geq \bar{\lambda}
$$

Since $\bar{\lambda}$ is the optimal value for the problem with proportional transaction costs we have the opposite inequality.

5. Incomplete observation. Usually investors do not have full information about factors having impact on the economy. It is due to the time needed to collect and process statistical data or simply due to inaccessibility of some information. Therefore, it is natural to extend our model to cover the case where a number of economic factors is either observable with delay and noise or not observable at all (see [22]). This general setting is obtained by considering an observation process whose dynamics depends on the factors. This is well-established in engineering applications, where the observation process usually consists of noisy and possibly biased readings of the variables. However, it was argued that in the financial context it is natural to assume that we have complete observation of a group of factors and the rest is not observable. It does not substantially change the reasoning but simplifies the notation.

Following the above remark assume that the space of economic factors $E$ is a direct sum of metric spaces $E^{1}, E^{2}$ with Borel $\sigma$-algebras $\mathcal{E}^{1}, \mathcal{E}^{2}$. Therefore, $Z(t)$ has a unique decomposition into $\left(Z^{1}(t), Z^{2}(t)\right)$. We shall treat $E^{1}$ as the observable part of the economic factor space and $Z^{1}(t)$ as the observable factor process. The process $Z^{2}(t)$ is the unobservable part of the factor process. We denote by $\mathcal{M}_{t}, \mathcal{Z}_{t}^{1}, \mathcal{Z}_{t}^{2}$ filtrations generated, respectively, by $\zeta(t), Z^{1}(t)$ and $Z^{2}(t)$. Filtration $\mathcal{Y}_{t}$ represents our observation and is generated by $\mathcal{M}_{t}$ and $\mathcal{Z}_{t}^{1}$. Although above filtrations depend on the initial value $\left(z_{1}, z_{2}\right)$ of the process $Z(t)$, we will omit this dependence in the notation. Due to restriction in the available information, we have to modify the set of admissible portfolios $\tilde{\mathcal{A}}$ : it consists of all admissible portfolios from $\mathcal{A}$ that make use of the information available in $\left(\mathcal{Y}_{t}\right)$, i.e. that are $\mathcal{Y}_{t}$-adapted. The goal of this section is to prove existence of an optimal strategy maximizing the functional

$$
J^{\pi_{-}, x_{-}, z^{1}, \rho}(\Pi)=\liminf _{T \rightarrow \infty} \frac{1}{T} \mathbb{E}^{z^{1}, \rho} \ln X_{-}^{\Pi}(T)
$$


over all strategies $\Pi \in \tilde{\mathcal{A}}$. Here $\left(z^{1}, \rho\right) \in E^{1} \times \mathcal{P}\left(Z^{2}\right)$ denotes the initial distribution of $\left(Z^{1}(t), Z^{2}(t)\right)$ and $\mathcal{P}\left(Z^{2}\right)$ stands for the space of probability measures on $\left(Z^{2}, \mathcal{E}^{2}\right)$, the a priori knowledge of the value $Z^{2}(0)$. The method of proof will be similar to that of Section 4. The following assumptions on the transition probabilities are required:

(B1) There exists a measure $\nu$ on $\mathbb{R}^{d}$ and a positive continuous bounded function $q: E^{1} \times E^{2} \times \mathbb{R}^{d} \rightarrow[0, \infty)$ such that for any $A \in \mathcal{B}\left(\mathbb{R}^{d}\right)$

$$
\mathbb{P}\left(\zeta(t+1) \in A \mid \mathcal{M}_{t} \vee \mathcal{Z}_{t+1}^{1} \vee \mathcal{Z}_{t+1}^{2}\right)=\int_{A} q\left(Z^{1}(t+1), Z^{2}(t+1), \tilde{r}\right) \nu(d \tilde{r})
$$

(B2) There exist a measure $p_{1}$ on $E^{1}$ and a positive continuous bounded function $\hat{p}_{1}: E^{1} \times E^{2} \times E^{1} \rightarrow(0, \infty)$ such that for any $A \in \mathcal{E}^{1}$ (recall that $P$ is a transition operator of $Z(t))$

$$
P\left(\left(z^{1}, z^{2}\right), A \times E^{2}\right)=\mathbb{P}^{z^{1}, z^{2}}\left(Z^{1}(1) \in A\right)=\int_{A} \hat{p}_{1}\left(z^{1}, z^{2}, z^{\prime}\right) p_{1}\left(d z^{\prime}\right) .
$$

(B3) There exist a measure $p_{2}$ on $E^{2}$ and a positive continuous function $\hat{p}_{2}: E^{1} \times$ $E^{2} \times E^{1} \times E^{2} \rightarrow(0, \infty)$ such that for any $A \in \mathcal{E}^{2}$

$$
\mathbb{P}\left\{Z^{2}(t+1) \in A \mid \mathcal{M}_{t} \vee \mathcal{Z}_{t+1}^{1} \vee \mathcal{Z}_{t}^{2}\right\}=\int_{A} \hat{p}_{2}\left(Z^{1}(t), Z^{2}(t), Z^{1}(t+1), z^{\prime}\right) p_{2}\left(d z^{\prime}\right)
$$

\section{Remarks.}

(1) The above assumptions are standard in the case of stochastic control with incomplete information (see [15], [21], [23]).

(2) Assumption (B2) is satisfied if the transition probabilities of the economic factor process $Z(t)$ are equivalent and enjoy densities (with respect to some probability measure) that depend continuously on all parameters.

(3) Let $E^{\xi}$ be a bounded open set in $\mathbb{R}^{d}$ and

$$
\zeta(z, \xi)=\left(\zeta^{1}(z, \xi), \ldots, \zeta^{d}(z, \xi)\right), \quad(z, \xi) \in\left(E, E^{\xi}\right) .
$$

Assume that $\xi$ has a continuous density $k(y)$ with respect to Lebesgue measure on $E^{\xi}$. If $\xi \mapsto \zeta(z, \xi)$ is a diffeomorphism for every $z \in E$ then assumption (B1) holds with $\nu$ being Lebesgue measure on $E^{\xi}$ and

$$
q\left(z_{1}, z_{2}, \tilde{r}\right)=k(g(z, \tilde{r})) \operatorname{det}\left(D_{\tilde{r}} g(z, \tilde{r})\right)
$$

where $g(z, \cdot)$ is the inverse function for $\zeta(z, \cdot)$. If $E^{\xi}$ is an unbounded open set (e.g. the whole space) we need to assume that $k(\cdot)$ and first derivatives of $g(z, \cdot)$ with respect to the second variable are uniformly bounded for every $z \in E$.

(4) Notice that in (B3) we do not assume boundedness of $\hat{p}_{2}$.

(5) Condition (B3) is slightly stronger than it is usually assumed. Instead we could use 
(B3') There exist a measure $p_{2}$ on $E^{2}$ and a positive continuous function $\hat{p}_{2}: E^{1} \times$ $E^{2} \times \mathbb{R}^{d} \times E^{1} \times E^{2} \rightarrow(0, \infty)$ such that for any $A \in \mathcal{E}^{2}$

$$
\mathbb{P}\left\{Z^{2}(t+1) \in A \mid \mathcal{M}_{t} \vee \mathcal{Z}_{t+1}^{1} \vee \mathcal{Z}_{t}^{2}\right\}=\int_{A} \hat{p}_{2}\left(Z^{1}(t), Z^{2}(t), \zeta(t), Z^{1}(t+1), z^{\prime}\right) p_{2}\left(d z^{\prime}\right)
$$

The only difference lies in $\hat{p}_{2}$ that in (B3') incorporates additionally the knowledge of the last price movement $\zeta(t)$. However, in our setting (B3) has a straightforward interpretation. Recall that in Section 3 we require that the economic factor process $Z(t)$ be itself Markovian. Moreover, the restriction to (B3) simplifies the notation. The results of this section can be easily generalized to cover (B3').

With the help of the above assumptions we are able to provide a Markovian representation of the filtering process

$$
\rho(t)(A)=\mathbb{P}^{z}\left(Z^{2}(t) \in A \mid \mathcal{M}_{t} \vee \mathcal{Z}_{t}^{1}\right), \quad A \in \mathcal{E}^{2} .
$$

Observe that $\rho(t)$ is a random variable with values in the space $\mathcal{P}\left(E^{2}\right)$ of probability measures on $\left(E^{2}, \mathcal{E}^{2}\right)$ equipped with the weak convergence topology.

LEMma 5.1. Under assumptions (B1)-(B3)

$$
\rho(t+1)(A)=M\left(Z^{1}(t), Z^{1}(t+1), \zeta(t+1), \rho(t)\right)(A), \quad A \in \mathcal{E}^{2},
$$

where

$$
M\left(z^{1}, \tilde{z}^{1}, \tilde{\varsigma}, \rho\right)(A)=\frac{\int_{E^{2}} \int_{A} q\left(\tilde{z}^{1}, \tilde{z}^{2}, \tilde{\varsigma}\right) \hat{p}_{2}\left(z^{1}, z^{2}, \tilde{z}^{1}, \tilde{z}^{2}\right) p_{2}\left(d \tilde{z}^{2}\right) \hat{p}_{1}\left(z^{1}, z^{2}, \tilde{z}^{1}\right) \rho\left(d z^{2}\right)}{\int_{E^{2}} \int_{E^{2}} q\left(\tilde{z}^{1}, \tilde{z}^{2}, \tilde{\varsigma}\right) \hat{p}_{2}\left(z^{1}, z^{2}, \tilde{z}^{1}, \tilde{z}^{2}\right) p_{2}\left(d \tilde{z}^{2}\right) \hat{p}_{1}\left(z^{1}, z^{2}, \tilde{z}^{1}\right) \rho\left(d z^{2}\right)} .
$$

Proof. The proof is rather standard and employs techniques from Lemma 1.1 in [15] or Lemma 1 in [21].

LEMMA 5.2. Under assumptions (B1)-(B3):

i) The process $\left(Z^{1}(t), \rho(t)\right)$ is Markovian with respect to the filtration $\mathcal{Y}_{t}$ with transition operator

$$
\begin{aligned}
& \dot{\Pi} F\left(z^{1}, \rho\right) \\
= & \int_{E^{2}} \int_{E} \int_{\mathbb{R}^{d}} F\left(\tilde{z}^{1}, M\left(z^{1}, \tilde{z}^{1}, \tilde{\varsigma}, \rho\right)\right) q\left(\tilde{z}^{1}, \tilde{z}^{2}, \tilde{\varsigma}\right) \nu(d \tilde{\varsigma}) P\left(\left(z^{1}, z^{2}\right), d \tilde{z}^{1} \times d \tilde{z}^{2}\right) \rho\left(d z^{2}\right)
\end{aligned}
$$

for a measurable bounded function $F: E^{1} \times \mathcal{P}\left(E^{2}\right) \rightarrow \mathbb{R}$. Moreover, $\dot{\Pi}\left(z^{1}, \rho\right)$ transforms the space of continuous, bounded functions into itself.

ii) The process $\left(\pi_{-}(t), Z^{1}(t), \rho(t)\right)$ is Markovian with respect to the filtration $\mathcal{Y}_{t}$ with transition operator

$$
\begin{array}{r}
\ddot{\Pi} F\left(\pi_{-}, z^{1}, \rho\right)=\int_{E^{2}} \int_{E} \int_{\mathbb{R}^{d}} F\left(\pi_{-} \diamond \tilde{\varsigma}, \tilde{z}^{1}, M\left(z^{1}, \tilde{z}^{1}, \tilde{\varsigma}, \rho\right)\right) q\left(\tilde{z}^{1}, \tilde{z}^{2}, \tilde{\varsigma}\right) \nu(d \tilde{\varsigma}) \\
P\left(\left(z^{1}, z^{2}\right), d \tilde{z}^{1} \times d \tilde{z}^{2}\right) \rho\left(d z^{2}\right)
\end{array}
$$


for a measurable bounded function $F: \mathcal{S} \times E^{1} \times \mathcal{P}\left(E^{2}\right) \rightarrow \mathbb{R}$. Here, $\pi_{-}(t)$ is the proportion process given by $\pi_{-}(t)=\pi_{-}(0) \diamond \zeta(1) \diamond \cdots \diamond \zeta(t)$. The transition operator $\ddot{\Pi}$ transforms the space of continuous, bounded functions into itself.

Proof. First we prove that $\left(Z^{1}(t), \rho(t)\right)$ is a Markov process with the above transition operator. Let $F$ be a measurable bounded function. For simplicity we shall skip the subscript in the expected value operator. Appropriate conditioning leads to the result:

$$
\begin{aligned}
& \mathbb{E}\left(F\left(Z^{1}(t+1), \rho(t+1)\right) \mid \mathcal{Y}_{t}\right) \\
& =\mathbb{E}\left(\mathbb{E}\left(F\left(Z^{1}(t+1), \rho(t+1)\right) \mid \mathcal{Y}_{t} \vee \zeta(t+1) \vee Z^{1}(t+1)\right) \mid \mathcal{Y}_{t}\right) \\
& =\mathbb{E}\left(F\left(Z^{1}(t+1), M\left(Z^{1}(t), Z^{1}(t+1), \zeta(t+1), \rho(t)\right)\right) \mid \mathcal{Y}_{t}\right) \\
& =\mathbb{E}\left(\int_{\mathbb{R}^{d}} F\left(Z^{1}(t+1), M\left(Z^{1}(t), Z^{1}(t+1), \tilde{\varsigma}, \rho(t)\right)\right)\right. \\
& \left.\quad \cdot q\left(Z^{1}(t+1), Z^{2}(t+1), \tilde{\varsigma}\right) \nu(d \tilde{\varsigma}) \mid \mathcal{Y}_{t}\right) \\
& =\ldots \\
& =\int_{E^{2}} \int_{E} \int_{\mathbb{R}^{d}} F\left(\tilde{z}^{1}, M\left(Z^{1}(t), \tilde{z}^{1}, \tilde{\varsigma}, \rho(t)\right)\right) q\left(\tilde{z}^{1}, \tilde{z}^{2}, \tilde{\varsigma}\right) \nu(d \tilde{\varsigma}) \\
& \cdot P\left(\left(Z^{1}(t), z^{2}\right), d \tilde{z}^{1} \times d \tilde{z}^{2}\right) \rho(t)\left(d z^{2}\right) .
\end{aligned}
$$

To show that $\left(z^{1}, \tilde{z}^{1}, \tilde{\varsigma}, \rho\right) \mapsto M\left(z^{1}, \tilde{z}^{1}, \tilde{\varsigma}, \rho\right)$ is continuous, it suffices to prove that for any $A \in \mathcal{E}^{2}$

$$
\left(z^{1}, \tilde{z}^{1}, \tilde{\varsigma}, \rho\right) \mapsto \int_{E^{2}} \int_{A} q\left(\tilde{z}^{1}, \tilde{z}^{2}, \tilde{\varsigma}\right) \hat{p}_{2}\left(z^{1}, z^{2}, \tilde{z}^{1}, \tilde{z}^{2}\right) p_{2}\left(d \tilde{z}^{2}\right) \hat{p}_{1}\left(z^{1}, z^{2}, \tilde{z}^{1}\right) \rho\left(d z^{2}\right)
$$

is continuous. By Scheffe's theorem (see [19])

$$
\left(z^{1}, z^{2}, \tilde{z}^{1}, \tilde{\varsigma}\right) \mapsto \int_{A} q\left(\tilde{z}^{1}, \tilde{z}^{2}, \tilde{\varsigma}\right) \hat{p}_{2}\left(z^{1}, z^{2}, \tilde{z}^{1}, \tilde{z}^{2}\right) p_{2}\left(d \tilde{z}^{2}\right) \hat{p}_{1}\left(z^{1}, z^{2}, \tilde{z}^{1}\right)
$$

is continuous. Another application of Scheffe's theorem completes the proof.

A similar argument yields continuity of the mapping

$$
\left(z^{1}, \tilde{z}^{1}, \tilde{z}^{2}, \rho\right) \mapsto \int_{\mathbb{R}^{d}} F\left(\tilde{z}^{1}, M\left(z^{1}, \tilde{z}^{1}, \tilde{\varsigma}, \rho\right)\right) q\left(\tilde{z}^{1}, \tilde{z}^{2}, \tilde{\varsigma}\right) \nu(d \tilde{\varsigma}) .
$$

By Feller property of $\left(Z^{1}(t), Z^{2}(t)\right)$ we have continuity of

$$
\left(z^{1}, z^{2}, \rho\right) \mapsto \int_{E} \int_{\mathbb{R}^{d}} F\left(\tilde{z}^{1}, M\left(z^{1}, \tilde{z}^{1}, \tilde{\varsigma}, \rho\right)\right) q\left(\tilde{z}^{1}, \tilde{z}^{2}, \tilde{\varsigma}\right) \nu(d \tilde{\varsigma}) P\left(\left(z^{1}, z^{2}\right), d \tilde{z}^{1} \times d \tilde{z}^{2}\right) .
$$

Finally, application of Scheffe's theorem completes the proof.

The other statement has an analogous proof. 
Lemma 5.1 can be generalized to obtain a formula for $\rho(t)$ given $\rho(0)$. First observe that $M\left(z^{1}, \tilde{z}^{1}, \tilde{\varsigma}, \rho\right)(A)=N\left(z^{1}, \tilde{z}^{1}, \tilde{\varsigma}, \rho\right)(A) / N\left(z^{1}, \tilde{z}^{1}, \tilde{\varsigma}, \rho\right)\left(E^{2}\right)$, where

$$
\begin{aligned}
& N\left(z^{1}, \tilde{z}^{1}, \tilde{\varsigma}, \rho\right)(A) \\
= & \int_{E^{2}} \int_{A} q\left(\tilde{z}^{1}, \tilde{z}^{2}, \tilde{\varsigma}\right) \hat{p}_{2}\left(z^{1}, z^{2}, \tilde{z}^{1}, \tilde{z}^{2}\right) p_{2}\left(d \tilde{z}^{2}\right) \hat{p}_{1}\left(z^{1}, z^{2}, \tilde{z}^{1}\right) \rho\left(d z^{2}\right), \quad A \in \mathcal{E}^{2} .
\end{aligned}
$$

Put

$$
N_{1}\left(Z^{1}(0), Z^{1}(1), \zeta(1), \rho\right)(A)=N\left(Z^{1}(0), Z^{1}(1), \zeta(1), \rho\right)(A)
$$

and, for $t \geq 2$,

$$
\begin{aligned}
& N_{t}\left(Z^{1}(0), \ldots, Z^{1}(t), \zeta(1), \ldots, \zeta(t), \rho\right)(A) \\
= & N\left(Z^{1}(t-1), Z^{1}(t), \zeta(t), N_{t-1}\left(Z^{1}(0), \ldots, Z^{1}(t-1), \zeta(1), \ldots, \zeta(t-1), \rho\right)\right)(A) .
\end{aligned}
$$

For $\left(z^{1}, \rho\right) \in E^{1} \times \mathcal{P}\left(E^{2}\right)$ we denote by $P\left(\left(z^{1}, \rho\right), \cdot\right)$ the operator

$$
\int_{E^{2}} P\left(\left(z^{1}, z^{2}\right), \cdot\right) \rho\left(d z^{2}\right)
$$

Easy calculations lead to the following lemma.

Lemma 5.3. i) $N_{t}(\cdots)\left(E^{2}\right)$ is a martingale with mean 1 with respect to the measure $P^{\left(z^{1}, \rho\right)}$ and filtration $\mathcal{Y}_{t}$.

ii) $\rho(t)=M_{t}\left(Z^{1}(0), \ldots, Z^{1}(t), \zeta(1), \ldots, \zeta(t), \rho(0)\right)$, where

$M_{t}\left(Z^{1}(0), \ldots, Z^{1}(t), \zeta(1), \ldots, \zeta(t), \rho\right)(A)=\frac{N_{t}\left(Z^{1}(0), \ldots, Z^{1}(t), \zeta(1), \ldots, \zeta(t), \rho\right)(A)}{N_{t}\left(Z^{1}(0), \ldots, Z^{1}(t), \zeta(1), \ldots, \zeta(t), \rho\right)\left(E^{2}\right)}$.

Now we shall construct such a measure that processes $Z^{1}(t), Z^{2}(t), \zeta(t)$ become independent and consist of i.i.d. random variables. For $\left(z^{1}, z^{2}\right) \in E^{1} \times E^{2}$ consider

$$
\begin{aligned}
& L_{t}^{\left(z^{1}, z^{2}\right)}=\prod_{s=1}^{t} q\left(Z^{1}(s), Z^{2}(s), \zeta(s)\right) \hat{p}_{1}\left(Z^{1}(s-1), Z^{2}(s-1), Z^{1}(s)\right) \\
& \hat{p}_{2}\left(Z^{1}(s-1), Z^{2}(s-1), Z^{1}(s), Z^{2}(s)\right) .
\end{aligned}
$$

By direct calculations $\Lambda_{t}^{\left(z^{1}, z^{2}\right)}=\left(L_{t}^{\left(z^{1}, z^{2}\right)}\right)^{-1}$ is a positive martingale with mean 1 with respect to measure $\mathbb{P}^{\left(z^{1}, z^{2}\right)}$ and filtration $\mathcal{F}_{t}$. Let $\mathbb{P}_{0}$ be a probability measure whose restrictions to $\mathcal{F}_{t}$ have density $\Lambda_{t}$ with respect to $\mathbb{P}^{\left(z^{1}, z^{2}\right)}$. The measure $\mathbb{P}_{0}$ may not be absolutely continuous with respect to $\mathbb{P}^{\left(z^{1}, z^{2}\right)}$, but its restrictions to $\mathcal{F}_{t}$ for finite $t$ are equivalent to $\mathbb{P}^{\left(z^{1}, z^{2}\right)}$. It can be easily calculated (see Lemma 1.8 in [15]) that with respect to measure $\mathbb{P}_{0}$ for any $t>0$ processes $Z^{1}(s), Z^{2}(s), \zeta(s), 1 \leq s \leq t$, are independent and consist of i.i.d. random variables with distributions $p_{1}, p_{2}, \nu$, respectively. The following lemma applies above results to the filtered process: 
LEMMA 5.4. For a measurable bounded function $f: E^{1} \times \mathcal{P}\left(E^{2}\right) \times\left(E^{1} \times \mathcal{P}\left(E^{2}\right) \times\right.$ $\left.\mathbb{R}^{d}\right)^{t} \rightarrow \mathbb{R}$ we have

$$
\begin{aligned}
\mathbb{E}^{\left(z^{1}, \rho\right)} f\left(Z^{1}(0), \rho(0), Z^{1}(1), \rho(1), \zeta(1), \ldots, Z^{1}(t), \rho(t), \zeta(t)\right) \\
=\mathbb{E}_{0}\left\{N_{t}\left(z^{1}, \tilde{z}_{1}^{1}, \ldots, \tilde{z}_{t}^{1}, \tilde{\zeta}_{1}, \ldots, \tilde{\zeta}_{t}, \rho\right)\left(E^{2}\right)\right. \\
\left.f\left(z^{1}, \rho, \tilde{z}_{1}^{1}, \tilde{\rho}_{1}, \tilde{\zeta}_{1}, \ldots, \tilde{z}_{t}^{1}, \tilde{\rho}_{t}, \tilde{\zeta}_{t}\right)\right\}
\end{aligned}
$$

where $\mathbb{E}_{0}$ is the expected value operator related to some measure $\mathbb{P}_{0}$ with respect to which $\tilde{z}_{s}^{1}, \tilde{\zeta}_{s}, s=0, \ldots, t$, are independent random variables with distributions $p_{1}, \nu$, respectively and $\tilde{\rho}_{s}=M_{s}\left(z^{1}, \tilde{z}_{1}^{1}, \ldots, \tilde{z}_{s}^{1}, \tilde{\zeta}_{1}, \ldots, \tilde{\zeta}_{s}, \rho\right), s=1, \ldots, t$.

Proof. By easy calculation we have

$$
\begin{aligned}
\mathbb{E}^{\left(z^{1}, \rho\right)} f(\cdots) & =\int_{E^{2}} \mathbb{E}^{\left(z^{1}, z^{2}\right)} f(\cdots) \rho\left(d z^{2}\right) \\
& =\int_{E^{2}} \mathbb{E}_{0} L_{t}^{\left(z^{1}, z^{2}\right)} f(\cdots) \rho\left(d z^{2}\right) \\
& =\int_{E^{2}} \mathbb{E}_{0}\left\{N_{t}(\cdots)\left(E^{2}\right) f(\cdots)\right\} \rho\left(d z^{2}\right)=\mathbb{E}_{0}\left\{N_{t}(\cdots)\left(E^{2}\right) f(\cdots)\right\}
\end{aligned}
$$

where $\mathbb{E}_{0}^{\left(z^{1}, z^{2}\right)}$ represents the expected value operator for $\mathbb{P}_{0}$. In the last equality, we can skip the integration with respect to $z^{2}$ since the integrand does not depend on $z^{2}$.

To formulate an analog of Theorem 3.1 we need two more assumptions. We shall use the notation from Lemma 5.4. For $z^{1}, \hat{z}^{1} \in E^{1}, \rho, \hat{\rho} \in \mathcal{P}\left(E^{2}\right), \epsilon \in(0,1)$ and $n \geq 1$ consider

$$
\begin{aligned}
& D_{n}^{\epsilon}\left(z^{1}, \rho, \hat{z}^{1}, \hat{\rho}\right) \\
= & \left\{\omega \in \Omega: \quad N_{n}\left(\hat{z}^{1}, \tilde{z}_{1}^{1}, \ldots, \tilde{z}_{n}^{1}, \tilde{\zeta}_{1}, \ldots, \tilde{\zeta}_{n}, \hat{\rho}\right) \geq \epsilon N_{n}\left(z^{1}, \tilde{z}_{1}^{1}, \ldots, \tilde{z}_{n}^{1}, \tilde{\zeta}_{1}, \ldots, \tilde{\zeta}_{n}, \rho\right)\right\}
\end{aligned}
$$

(B4) $\exists_{n} \exists_{\epsilon \in(0,1), \delta>0}$ such that for $z^{1}, \hat{z}^{1} \in E^{1}, \rho, \hat{\rho} \in \mathcal{P}\left(E^{2}\right)$

$$
\mathbb{E}_{0}\left\{1_{D_{n}^{\epsilon}\left(z^{1}, \rho, \hat{z}^{1}, \hat{\rho}\right)} N_{n}\left(z^{1}, \tilde{z}_{1}^{1}, \ldots, \tilde{z}_{n}^{1}, \tilde{\zeta}_{1}, \ldots, \tilde{\zeta}_{n}, \rho\right)\right\} \geq \delta
$$

(B5) $E^{2}$ is compact.

THEOREM 5.5. Under assumptions (A1)-(A2), (A4), (B1)-(B5) there exists a measurable function $p: \mathcal{S} \times(0, \infty) \times E^{1} \times \mathcal{P}\left(E^{2}\right) \rightarrow \mathcal{S}$, a constant $\lambda$ and a measurable set $I \subseteq \mathcal{S} \times(0, \infty) \times E^{1} \times \mathcal{P}\left(E^{2}\right)$ such that

$$
\lambda=J^{\pi_{-}, x_{-}, z^{1}, \rho}\left(\Pi^{*}\right)=\sup _{\Pi \in \tilde{\mathcal{A}}} J^{\pi_{-}, x_{-}, z^{1}, \rho}(\Pi),
$$


where the optimal portfolio $\Pi^{*}=\left(\left(\pi_{1}^{*}, \tau_{1}^{*}\right),\left(\pi_{2}^{*}, \tau_{2}^{*}\right), \ldots\right)$ is given by the formulas

$$
\begin{aligned}
& \tau_{1}^{*}=\inf \left\{t \geq 0: \quad\left(\pi_{-}(t), X_{-}(t), Z^{1}(t), \rho(t)\right) \in I\right\}, \\
& \tau_{k+1}^{*}=\inf \left\{t>\tau_{k}^{*}: \quad\left(\pi_{-}(t), X_{-}(t), Z^{1}(t), \rho(t)\right) \in I\right\}, \\
& \pi_{k}^{*}=p\left(\pi_{-}\left(\tau_{k}^{*}\right), X_{-}\left(\tau_{k}^{*}\right), Z^{1}\left(\tau_{k}^{*}\right), \rho\left(\tau_{k}^{*}\right)\right) .
\end{aligned}
$$

Proof. First observe that by appropriate conditioning we obtain

$$
\begin{aligned}
J_{\beta}^{\pi_{-}, x_{-}, z^{1}, \rho}=\liminf _{T \rightarrow \infty} \frac{1}{T}\{ & \sum_{t=0}^{T-1} \mathbb{E}^{z^{1}, \rho} g\left(\pi(t), Z^{1}(t), \rho(t)\right) \\
& \left.+\sum_{k=1}^{\infty} \mathbb{E}^{z^{1}, \rho}\left\{1_{\tau_{k}<T} \ln e\left(\pi_{-}\left(\tau_{k}\right), \pi_{k}, X_{-}\left(\tau_{k}\right)\right)\right\}\right\},
\end{aligned}
$$

where

$$
g\left(\pi, z^{1}, \rho\right)=\int_{E^{2}} h\left(\pi, z^{1}, z^{2}\right) \rho\left(d z^{2}\right) .
$$

Notice that $g$ is continuous and bounded by boundedness and continuity of $h$. Now, the proof follows by the same consideration as that in the proof of Theorem 3.1. Under assumption (B5) the space $\mathcal{P}\left(E^{2}\right)$ is compact. Therefore, $\mathcal{S} \times(0, \infty) \times E^{1} \times \mathcal{P}\left(E^{2}\right)$ is a locally compact separable metric space, which is needed for validity of Lemma 4.9 and Lemma 4.10. Since we do not have (A3), Lemma 4.4 requires a new proof, which we shall present below. To clarify the notation let

$J_{\beta}^{\pi_{-}, z^{1}, \rho}(\Pi)=\mathbb{E}^{\left(z^{1}, \rho\right)}\left\{\sum_{t=1}^{\infty} \beta^{t} g\left(\pi(t), Z^{1}(t), \rho(t)\right)+\sum_{k=1}^{\infty} \beta^{\tau_{k}} \ln e\left(\pi_{-}\left(\tau_{k}\right), \pi_{k}\right)\right\}, \beta \in(0,1)$.

for $\Pi \in \tilde{\mathcal{A}}$. It is a discounted functional related to the problem with only proportional transaction costs. Denote by $v_{\beta}\left(\pi_{-}, z^{1}, \rho\right)$ the value function corresponding to this functional. By Theorem 4.2 it is bounded and continuous. We shall, however, prove that it is bounded uniformly in $\beta$ with respect to the span seminorm.

For a bounded measurable function $f: \mathcal{P}\left(E^{2}\right) \rightarrow \mathbb{R}, \mu \in M^{+}\left(\mathcal{E}^{2}\right)$ (the space of non-negative non-null finite measures on $\left.\mathcal{E}^{2}\right)$, define an operator

$$
S f(\mu)=\mu\left(E^{2}\right) f\left(\frac{\mu}{\mu\left(E^{2}\right)}\right) .
$$

In order to simplify the notation whenever $f$ depends on more than one variable the operator $S f$ is meant to act only on the measure-valued variable. Notice that by Lemma 5.4

$$
E^{\left(z^{1}, \rho\right)} f\left(Z^{1}(t), \rho(t)\right)=\mathbb{E}_{0} S f\left(Z^{1}(t), N_{t}\left(z^{1}, \tilde{z}_{1}^{1}, \ldots, \tilde{z}_{t}^{1}, \tilde{\zeta}_{1}, \ldots, \tilde{\zeta}_{t}, \rho\right)\right)
$$


LEMMA 5.6. i) The value function $v_{\beta}\left(\pi_{-}, z^{1}, \rho\right)$ is concave with respect to the third variable, i.e. for $\rho, \hat{\rho} \in \mathcal{P}\left(E^{2}\right)$ and $\lambda \in(0,1)$

$$
v_{\beta}\left(\pi_{-}, z^{1}, \lambda \rho+(1-\lambda) \hat{\rho}\right) \geq \lambda v_{\beta}\left(\pi_{-}, z^{1}, \rho\right)+(1-\lambda) v_{\beta}\left(\pi_{-}, z^{1}, \hat{\rho}\right) .
$$

ii) If $f: \mathcal{P}\left(E^{2}\right) \rightarrow \mathbb{R}$ is a concave bounded measurable function, then $S f$ is concave.

Proof. We shall present only a sketch of (i) (a full proof can be found in [21] Theorem 1). First we show that for a bounded measurable $F: \mathcal{S} \times E^{1} \times \mathcal{P}\left(E^{2}\right) \rightarrow \mathbb{R}$ concave with respect to the third argument,

$$
\rho \mapsto \ddot{\Pi} F\left(\pi_{-}, z^{1}, \rho\right)
$$

is concave (the operator $\ddot{\Pi}$ is defined in Lemma 5.2). Then, using a notation of Theorem 4.2 , we show by induction on $k$ that $v_{\beta}^{k}\left(\pi_{-}, z^{1}, \rho\right)$ is concave with respect to the third argument.

The proof of (ii) is basic and can be found in [5] Lemma 2 .

To show a counterpart of Lemma 4.4 we use an approach from Section 5 of [6]. Namely,

$$
\begin{aligned}
& v_{\beta}\left(\pi_{-}, z^{1}, \rho\right)-v_{\beta}\left(\hat{\pi}_{-}, \hat{z}^{1}, \hat{\rho}\right) \\
& \leq n\|g\|_{s p}-(n+2) \ln \underline{e} \\
& \quad+\left(\mathbb{E}^{\left(z^{1}, \rho\right)} v_{\beta}\left(\pi^{*}, Z^{1}(t), \rho(t)\right)-\mathbb{E}^{\left(\hat{z}^{1}, \hat{\rho}\right)} v_{\beta}\left(\pi^{*}, \hat{Z}^{1}(t), \hat{\rho}(t)\right)\right)
\end{aligned}
$$

for some $\pi^{*} \in \mathcal{S}$. Let $h_{\beta}\left(\pi_{-}, z^{1}, \rho\right)=v_{\beta}\left(\pi_{-}, z^{1}, \rho\right)-\inf v_{\beta}\left(\pi_{-}, z^{1}, \rho\right)$. Hence $\left\|v_{\beta}\right\|_{s p}=$ $\left\|h_{\beta}\right\|$, where $\|\cdot\|$ is the supremum norm. Using assumption (B4) and Lemma 5.4 we obtain

$$
\begin{aligned}
& \mathbb{E}^{\left(z^{1}, \rho\right)} v_{\beta}\left(\pi^{*}, Z^{1}(t), \rho(t)\right)-\mathbb{E}^{\left(\hat{z}^{1}, \hat{\rho}\right)} v_{\beta}\left(\pi^{*}, \hat{Z}^{1}(t), \hat{\rho}(t)\right) \\
\leq & \mathbb{E}_{0}\left\{1 _ { D _ { n } ^ { \epsilon } ( z ^ { 1 } , \rho , \hat { z } ^ { 1 } , \hat { \rho } ) } \left(S h_{\beta}\left(\pi^{*}, \tilde{z}_{t}^{1}, N_{n}\left(z^{1}, \tilde{z}_{1}^{1}, \ldots, \tilde{z}_{n}^{1}, \tilde{\zeta}_{1}, \ldots, \tilde{\zeta}_{n}, \rho\right)\right)\right.\right. \\
& \left.\left.-S h_{\beta}\left(\pi^{*}, \tilde{z}_{t}^{1}, N_{n}\left(\hat{z}^{1}, \tilde{z}_{1}^{1}, \ldots, \tilde{z}_{n}^{1}, \tilde{\zeta}_{1}, \ldots, \tilde{\zeta}_{n}, \hat{\rho}\right)\right)\right)\right\} \\
& +\left\|h_{\beta}\right\| \mathbb{E}_{0}\left\{\left(1-1_{D_{n}^{\epsilon}\left(z^{1}, \rho, \hat{z}^{1}, \hat{\rho}\right)}\right) N_{n}\left(z^{1}, \tilde{z}_{1}^{1}, \ldots, \tilde{z}_{n}^{1}, \tilde{\zeta}_{1}, \ldots, \tilde{\zeta}_{n}, \rho\right)\right\} .
\end{aligned}
$$

On $D_{n}^{\epsilon}\left(z^{1}, \rho, \hat{z}^{1}, \hat{\rho}\right)$ we have

$$
N_{n}\left(\hat{z}^{1}, \tilde{z}_{1}^{1}, \ldots, \tilde{z}_{n}^{1}, \tilde{\zeta}_{1}, \ldots, \tilde{\zeta}_{n}, \hat{\rho}\right)=\epsilon N_{n}\left(z^{1}, \tilde{z}_{1}^{1}, \ldots, \tilde{z}_{n}^{1}, \tilde{\zeta}_{1}, \ldots, \tilde{\zeta}_{n}, \rho\right)+(1-\epsilon) \mu
$$

for some $\mu \in M^{+}\left(\mathcal{E}^{2}\right)$. By Lemma $5.3 \mu$ is not null. Hence by Lemma 5.6 we have

$$
\begin{aligned}
& S h_{\beta}\left(\pi^{*}, \tilde{z}_{t}^{1}, N_{n}\left(\hat{z}^{1}, \tilde{z}_{1}^{1}, \ldots, \tilde{z}_{n}^{1}, \tilde{\zeta}_{1}, \ldots, \tilde{\zeta}_{n}, \hat{\rho}\right)\right) \\
& \geq \epsilon h_{\beta}\left(\pi^{*}, \tilde{z}_{t}^{1}, N_{n}\left(z^{1}, \tilde{z}_{1}^{1}, \ldots, \tilde{z}_{n}^{1}, \tilde{\zeta}_{1}, \ldots, \tilde{\zeta}_{n}, \rho\right)\right)+(1-\epsilon) S h_{\beta}\left(\pi^{*}, \tilde{z}_{t}^{1}, \mu\right) \\
& \geq \epsilon h_{\beta}\left(\pi^{*}, \tilde{z}_{t}^{1}, N_{n}\left(z^{1}, \tilde{z}_{1}^{1}, \ldots, \tilde{z}_{n}^{1}, \tilde{\zeta}_{1}, \ldots, \tilde{\zeta}_{n}, \rho\right)\right) .
\end{aligned}
$$


Consequently, by Lemma 5.3 we have

$$
\begin{aligned}
\mathbb{E}^{\left(z^{1}, \rho\right)} v_{\beta}\left(\pi^{*}, Z^{1}(t), \rho(t)\right) & -\mathbb{E}^{\left(\hat{z}^{1}, \hat{\rho}\right)} v_{\beta}\left(\pi^{*}, \hat{Z}^{1}(t), \hat{\rho}(t)\right) \\
& \leq(1-\epsilon)\left\|h_{\beta}\right\| \delta+\left\|h_{\beta}\right\|(1-\delta)=\left\|h_{\beta}\right\|(1-\epsilon \delta) .
\end{aligned}
$$

We insert this estimate in (31) to obtain

$$
\left\|v_{\beta}\right\|_{s p} \leq n\|g\|_{s p}-(n+2) \ln \underline{e}+\left\|v_{\beta}\right\|_{s p}(1-\epsilon \delta) .
$$

Hence, we have

$$
\left\|v_{\beta}\right\|_{s p} \leq \frac{n\|g\|_{s p}-(n+2) \ln \underline{e}}{\epsilon \delta},
$$

which completes the proof of the counterpart of Lemma 4.4.

\section{Remarks.}

(1) If in place of (B4) we had

$$
\sup _{z^{1}, \hat{z}^{1} \in E^{1}} \sup _{\rho, \hat{\rho} \in \mathcal{P}\left(E^{2}\right)} \sup _{B \in \mathcal{E}^{1} \otimes \mathcal{B}\left(\mathcal{P}\left(E^{2}\right)\right)}\left(\Pi^{n}\left(z^{1}, \rho\right) 1_{B}-\Pi^{n}\left(\hat{z}^{1}, \hat{\rho}\right) 1_{B}\right)=\kappa<1
$$

for some $n \geq 1$, the proof of Theorem 5.5 would be significantly shorter. Condition (32) reads exactly as (A3) for the reformulated optimization problem (30). Therefore, Theorem 3.1 could be directly applied. We should stress, however, that (32) is very restrictive and is not satisfied in most applications.

(2) Assumption (B5) guarantees that $\mathcal{P}\left(E^{2}\right)$ is locally compact, which is needed for existence of Borel measurable selectors used in the proof. Relaxation of this condition requires use of universally measurable selectors and substantially enlarges the space of admissible portfolios.

(3) Observe that if $\mathbb{P}_{0}\left(D_{n}^{\epsilon}\left(z^{1}, \rho, \hat{z}^{1}, \hat{\rho}\right)\right)=1$, assumption (B4) is satisfied with $\delta=1$. This covers the case studied in [5] Section 3, where

$$
\begin{gathered}
\inf _{z^{1}, \tilde{z}^{1}, \hat{z}^{1} \in E^{1}} \inf _{z^{2}, \tilde{z}^{2}, \hat{z}^{2} \in E^{2}} \frac{\hat{p}_{2}\left(z^{1}, z^{2}, \tilde{z}^{1}, \tilde{z}^{2}\right)}{\hat{p}_{2}\left(\hat{z}^{1}, \hat{z}^{2}, \tilde{z}^{1}, \tilde{z}^{2}\right)}=\lambda_{2}>0, \\
\inf _{z^{1}, \tilde{z}^{1}, \hat{z}^{1} \in E^{1}} \inf _{z^{2}, \hat{z}^{2} \in E^{2}} \frac{\hat{p}_{1}\left(z^{1}, z^{2}, \tilde{z}^{1}\right)}{\hat{p}_{1}\left(\hat{z}^{1}, \hat{z}^{2}, \tilde{z}^{1}\right)}=\lambda_{1}>0 .
\end{gathered}
$$

Then given $z^{1}, \hat{z}^{1}, \rho, \hat{\rho}$

$$
N_{1}\left(\hat{z}^{1}, \tilde{z}_{1}^{1}, \tilde{\zeta}_{1}, \hat{\rho}\right) \geq \lambda_{1} \lambda_{2} N_{1}\left(z^{1}, \tilde{z}_{1}^{1}, \tilde{\zeta}_{1}, \rho\right) \quad \text { for all } \tilde{z}_{1}^{1} \in E^{2}, \tilde{\zeta}_{1} \in \mathbb{R}^{d}
$$




\section{REFERENCES}

[1] M. Akian, A. Sulem, And M. TAKSAR, Dynamic optimization of long term growth rate for a portfolio with transaction costs - the logarithmic utility case. Math. Finance 11:2(2001), pp. $153-188$.

[2] A. Arapostathis et AL, Discrete-time controlled Markov processes with average cost criterion: a survey. SIAM J. Control Optim, 31:2(1993), pp. 282 - 344.

[3] T. R. Bielecki And S. R. Pliska, Risk Sensitive Dynamic Asset Management. Appl. Math. Optim. 37(1999), pp. 337 - 360.

[4] T. R. Bielecki, S. R. Pliska, And M. Sherris, Risk sensitive asset allocation. J. Econ. Dyn. Control 24(2004), pp. 1145-1177.

[5] G. B. Di Masi And L. Stettner, Risk-sensitive control of discrete time partially observed Markov processes with infinite horizon. Stoch \& Stoch Reports 67(1999), pp. 309-322.

[6] G. B. Di Masi And L. Stettner, Ergodicity of filtering process by vanishing discount approach. submitted to Systems and Control Lett. 2006.

[7] J. L. Doob, (1953) Stochastic Processes. Wiley.

[8] T. Goll and J. Kallsen, A Complete Explicit Solution to the Log-Optimal Portfolio Problem. Ann. Applied Prob. 13(2003), pp. 774-799.

[9] O. Hernandez-Lerma and J. B. Lasserre, Discrete-Time Markov Control Processes. Springer, 1996.

[10] O. Hernandez-Lerma and J. B. Lasserre, Further Topics on Discrete-Time Markov Control Processes. Springer, 1999.

[11] G. Iyengar, Universal investment in markets with transaction costs. Math. Finance 15:2(2005), pp. 359-371.

[12] R. Korn And M. SCHÄL, On value preserving and growth optimal portfolios. Math. Meth. Oper. Res. 50(1999), pp. 189-218.

[13] J. Palczewski and L. Stettner, Impulsive control of portfolios. to appear in Appl. Math. Optim. 2005.

[14] E. Platen, A Benchmark Approach to Finance. Math. Finance 16(2006), pp. 131-151.

[15] W. J. Runggaldier and L. Stettner, Approximations of Discrete Time Partially Observed Control Problems. Giardini Editori, Pisa, 1994.

[16] R. Serfozo, Convergence of Lebesgue integrals with varying measures. Sankhya Ser.A 44:3(1982), pp. 380 - 402.

[17] M. SснӓL, A Selection Theorem for Optimisation Problems. Arch. Math. 25(1975), pp. 219-224.

[18] M. SсHёL, Average optimality in dynamic programming with general state space. Math. Oper. Res. 18:1(1993), pp. 163 - 172.

[19] H. Scheffe, A useful convergence theorem for probability distributions. Ann. Math. Statist. 18(1947), pp. 434-438.

[20] L. Stettner, On impulsive control with long run average cost criterion. Studia Mathematica 76:3(1983), pp. 279 - 298.

[21] L. StetTNeR, Ergodic control of partially observed Markov processes with equivalent transition probabilities. Applicationes Mathematicae 22:1(1993), pp. 25 - 38.

[22] L. Stettner, Risk Sensitive Portfolio Optimization with Completely and Partially Observed Factors. IEEE Trans. Automat. Control 49(2004), pp. 457 - 464.

[23] L. Stettner, Discrete Time Risk Sensitive Portfolio Optimization with Consumption and Proportional Transaction Costs. Applicationes Mathematicae 32:4(2005), pp. 395 - 404. 